\title{
Autism-linked dopamine transporter mutation alters striatal dopamine neurotransmission and dopamine- dependent behaviors
}

\author{
Gabriella E. DiCarlo, ${ }^{1}$ Jenny I. Aguilar, ${ }^{2,3}$ Heinrich J.C. Matthies, ${ }^{3}$ Fiona E. Harrison, ${ }^{1,4}$ Kyle E. Bundschuh, ${ }^{1}$ Alyssa West, ${ }^{5}$ \\ Parastoo Hashemi, ${ }^{5}$ Freja Herborg, ${ }^{6}$ Mattias Rickhag, ${ }^{6}$ Hao Chen, ${ }^{7}$ Ulrik Gether, ${ }^{6}$ Mark T. Wallace, ${ }^{1,8}$ and Aurelio Galli ${ }^{3}$ \\ 'Vanderbilt University Brain Institute, Nashville, Tennessee, USA. ²Department of Pharmacology, Vanderbilt University, Nashville, Tennessee, USA. ${ }^{3}$ Department of Surgery, University of Alabama at \\ Birmingham, Birmingham, Alabama, USA. ${ }^{4}$ Department of Medicine, Vanderbilt University Medical Center, Nashville, Tennessee, USA. ${ }^{5}$ Department of Chemistry and Biochemistry, University of South \\ Carolina, Columbia, South Carolina, USA. ${ }^{6}$ Molecular Neuropharmacology and Genetics Laboratory, Department of Neuroscience, Faculty of Health and Medical Sciences, University of Copenhagen, \\ Copenhagen, Denmark. ${ }^{7}$ DRI Biosciences Corp., Frederick, Maryland, USA. ${ }^{8}$ Department of Hearing and Speech Sciences, Vanderbilt University, Nashville, Tennessee, USA.
}

\begin{abstract}
The precise regulation of synaptic dopamine (DA) content by the DA transporter (DAT) ensures the phasic nature of the DA signal, which underlies the ability of DA to encode reward prediction error, thereby driving motivation, attention, and behavioral learning. Disruptions to the DA system are implicated in a number of neuropsychiatric disorders, including attention deficit hyperactivity disorder (ADHD) and, more recently, autism spectrum disorder (ASD). An ASD-associated de novo mutation in the SLC6A3 gene resulting in a threonine-to-methionine substitution at site 356 (DAT T356M) was recently identified and has been shown to drive persistent reverse transport of DA (i.e., anomalous DA efflux) in transfected cells and to drive hyperlocomotion in Drosophila melanogaster. A corresponding mutation in the leucine transporter, a DAT-homologous transporter, promotes an outward-facing transporter conformation upon substrate binding, a conformation possibly underlying anomalous DA efflux. Here, we investigated in vivo the impact of this ASD-associated mutation on DA signaling and ASD-associated behaviors. We found that mice homozygous for this mutation displayed impaired striatal DA neurotransmission and altered DA-dependent behaviors that correspond with some of the behavioral phenotypes observed in ASD.
\end{abstract}

\section{Introduction}

The dopamine (DA) system is a critical regulator of motor activity, motivation, attention, and reward processing (1-4). Dopaminergic projections originating from the substantia nigra and ventral tegmental area terminate in the striatum, where they function to regulate ongoing activity and gate motor function and influence thalamocortical signaling $(5,6)$. Given the integral role of this neurotransmitter system in critical brain functions, it is not surprising that dysregulation of the dopaminergic system has been implicated in a number of neuropsychiatric disorders, including Parkinson's disease (7), substance use disorder (8), bipolar disorder (BPD) (9), attention deficit hyperactivity disorder (ADHD) (10), and recently, autism spectrum disorder (ASD) (11-14). ASD is a neuropsychiatric disorder characterized by impairments in social communication and interaction and by patterns of restricted and repetitive behaviors, interests, or activities (15). As previous work has demonstrated that features of ASD may be due to or exacerbated by abnormal DA signaling

Authorship note: UG, MTW, and AG contributed equally to this work. Conflict of interest: $\mathrm{HC}$ is the president and CSO of DRI Biosciences Corporation that is developing treatments for neurological disorders including Fragile X Syndrome and Autism Spectrum Disorder.

Copyright: @ 2019, American Society for Clinical Investigation.

Submitted: January 14, 2019; Accepted: May 10, 2019; Published: July 22, 2019.

Reference information: J Clin Invest. 2019;129(8):3407-3419.

https://doi.org/10.1172/JCI127411.
$(16,17)$, the study of dopaminergic dysfunction as it relates to this disorder is vitally important.

The DA transporter (DAT), a presynaptic membrane protein found in dopaminergic terminals, regulates the temporal and spatial availability of DA (18) by rapidly clearing released DA from the synapse. The tight regulation of synaptic DA by DAT fine-tunes the phasic nature of DA signaling and thus the coding of both salience (19) and a quantitative reward prediction error signal (4, 20), which are critical for synaptic plasticity, reward processing, and behavioral learning $(21,22)$. It is not surprising, therefore, that genetic variants in the DAT gene have been linked to a number of neuropsychiatric disorders, including $\operatorname{ADHD}(23,24)$ and $\operatorname{ASD}(11$, 12) as well as neurological conditions such as Parkinson's disease (25) and parkinsonism-dystonia (26). Changes in the development of the striatum, a brain region enriched with DA projections and DAT expression, are associated with repetitive behaviors in autism. Disruptions in social-reward processing in the striatum (27) have also been found in individuals with ASD, and impairments in prediction and predictive coding have been suggested as causative factors in the clinical manifestation of ASD (28). Taken together, this evidence suggests that DAT dysfunction leading to altered dopaminergic signaling could support many aspects of the altered behaviors associated with neuropsychiatric disorders, such as ASD and ADHD.

Until recently, studies of DAT dysfunction in neuropsychiatric disorders and its downstream consequences for behaviors have been limited to DAT knockout (KO) (18), DAT heterozygous KO 
A

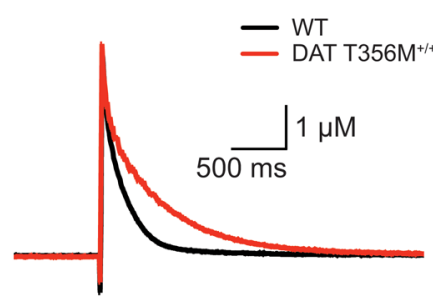

C

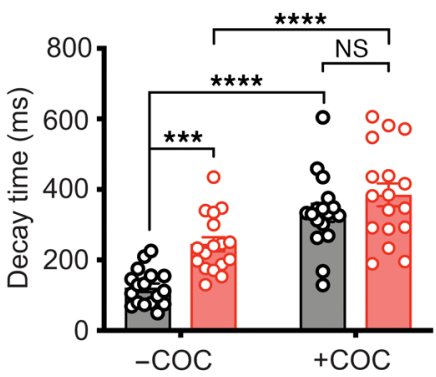

B

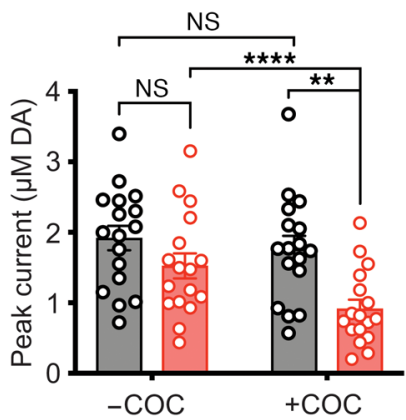

D

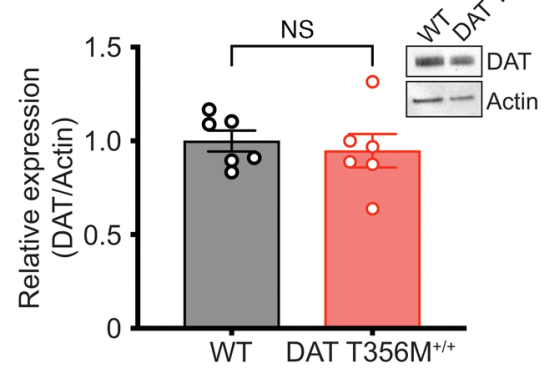

Figure 1. The DAT T356M mutation impairs striatal DA reuptake while maintaining normal DAT expression. (A) Stimulated dopaminergic current recorded (using carbon fiber amperometry) from acute striatal slices of WT (black) and DAT T356M+/+ (red) mice. (B) Peak dopaminergic current recorded from acute striatal slices of WT (black) and DAT T356M ${ }^{+/+}$(red) mice. There was no significant difference in the peak DA released between WT and DAT T356M ${ }^{+/+}$ mice at baseline (-cocaine [-COC]: WT $=1.921 \pm 0.17 \mu \mathrm{M}$; DAT T356M ${ }^{+/+}=1.526 \pm 0.18 \mu \mathrm{M} ; n=17$ $P=0.1908$, 2-way ANOVA). With addition of cocaine, peak DA released decreased significantly in DAT T356M ${ }^{+/+}$striatum, but not WT striatum $\left(+\mathrm{COC}: \mathrm{WT}=1.766 \pm 0.19 \mu \mathrm{M}\right.$; DAT T356M $\mathrm{M}^{+/+}=$ $0.916 \pm 0.13 \mu \mathrm{M} ; n=17 ; P=0.23$ and $P=<0.001$, respectively, 2-way ANOVA). ${ }^{* * *} P<0.0001$; ${ }^{* *} P=0.0013$ (C) Decay time $\left(\mathrm{t}_{80}-\mathrm{t}_{20}\right)$ of the dopaminergic signal recorded in acute striatal slices of WT (black) and DAT T356M ${ }^{+/+}$(red) mice. The decay time was significantly longer at baseline in DAT T356M ${ }^{+/+}$striatum when compared with WT striatum (-COC: WT $=121.5 \pm 12.39 \mathrm{~ms}$; DAT T356M $\mathrm{M}^{+/+}=244.9 \pm 19.83 ; n=17 ; P=0.001$, 2-way ANOVA). Addition of cocaine increased decay time of the dopaminergic signal in both WT and DAT T356M ${ }^{+/+}$mice $(+C O C:$ WT $=334.1$ $\pm 25.82 \mathrm{~ms} ;$ DAT T356M $\mathrm{M}^{+/+}=384.9 \pm 32.21 \mathrm{~ms} ; n=17 ; P=\langle 0.001 P=$ and 0.006 , respectively, 2-way ANOVA followed by Šidák's multiple comparisons test), as expected, and confirming the identity of the current as dopaminergic. ${ }^{* * *} P=0.001 ;{ }^{* * *} P<0.0001$ (D) Immunoblotting for DAT showed no difference in DAT expression in the striatum between WT and DAT T356M ${ }^{+/+}$ mice $(n=6 ; P=0.877$, 2-tailed $t$ test)

(29), and DAT knockdown mice (30) as well as DAT knockin mice expressing a mutant incapable of binding synaptic scaffold proteins (31). DAT KO mice exhibit reduced DA levels, increased extracellular DA, hyperactivity, and loss of sensitivity to cocaine and amphetamine (18). In comparison, DAT knockdown mice exhibit hyperactivity in novel environments, which is inhibited by amphetamine (30), while DAT heterozygous KO animals do not exhibit spontaneous hyperlocomotion (29). While the present genetic mouse models have been instrumental in our understanding of the biological and behavioral importance of DAT function, these models are less representative of known human genetic mutations in DAT and thus have more limited applicability to human disease.

More recent work has identified SNPs of DAT in individuals with ASD and associated comorbidities $(11,12,23)$. One such polymorphism is the substitution DAT Ala559Val. This mutation was identified in 2 individuals with ADHD (32), 2 individuals with ASD (12), and 1 with BPD (33). In vitro, this mutation was shown to promote DAT-mediated leak of cytoplasmic DA (34). While homozygous Ala559Val mice do exhibit increased darting speed upon imminent handling, they do not replicate most of the hallmark clinical features associated with the conditions in which this mutation was identified (35). Specifically, they do not exhibit spontaneous locomotor hyperactivity or stereotypies (35), as might be expected in ADHD and ASD, respectively.

Here we investigate what we believe is the first de novo ASD-associated mutation identified in DAT, a threonine-to-methionine substitution at site 356 (DAT T356M) (36), using a genetically modified knockin mouse model. This mutation, identified in an individual with ASD (for patient genetic information and clinical evaluation, see supplemental material for Hamilton et al.; ref. 37), is positioned in the seventh transmembrane domain of DAT near the ion-binding site (38). In vitro work has demonstrated that this mutation drives reduced DA reuptake as well as anomalous DA efflux (37) and significantly reduces the affinity of the transporter for cocaine and methylphenidate (suggesting an important role for T356 in inhibitor binding) (38). Models based on the bacterial homologous transporter LeuT predict that the T356M mutation promotes an outwardfacing transporter conformation when substrate is bound. In Drosophila melanogaster, the T356M variant has been shown to generate hyperlocomotion (37), but the impact of this mutation on DA homeostasis and DA-dependent behaviors in the mammalian brain has not been investigated. Here, we demonstrate that DAT T356M homozygous (DAT $\left.\mathrm{T} 356 \mathrm{M}^{+/+}\right)$mice display profound impairments in DA neurotransmission and clearance. These impairments translate to severe alterations of specific behaviors, which closely resemble those seen in the ASD clinical phenotype. While this work provides a better understanding of DAT biology in vivo, it also defines the impact of DAT dysregulation on specific behaviors and provides insight into the potential molecular mechanisms underlying the etiology of ASD and its comorbidities.

\section{Results}

DAT $T 356 \mathrm{M}^{+/+}$mice display impaired DA clearance while maintaining normal DAT expression. DAT T356 $\mathrm{M}^{+/}$and DAT T356 $\mathrm{M}^{+/+}$mice were generated as described in Methods. No significant differences were observed between WT and DAT T356 $\mathrm{M}^{+/-}$mice among all behavioral phenotypes analyzed in this study (see below). Therefore, electrophysiological and biochemical experiments were performed on WT and DAT T356 $\mathrm{M}^{+/+}$animals. We first sought to determine whether the T356M mutation, by promoting anomalous DA efflux, affects striatal DA clearance. We performed ex vivo chronoamperometry to measure electrically stimulated DA release and clearance in striatal slices (Figure 1A). We quantified peak DA release (Figure 1B) and the decay time of the amperometric current (Figure 1C). Peak DA release did not differ between WT and DAT T356 $\mathrm{M}^{+/+}$ 
A

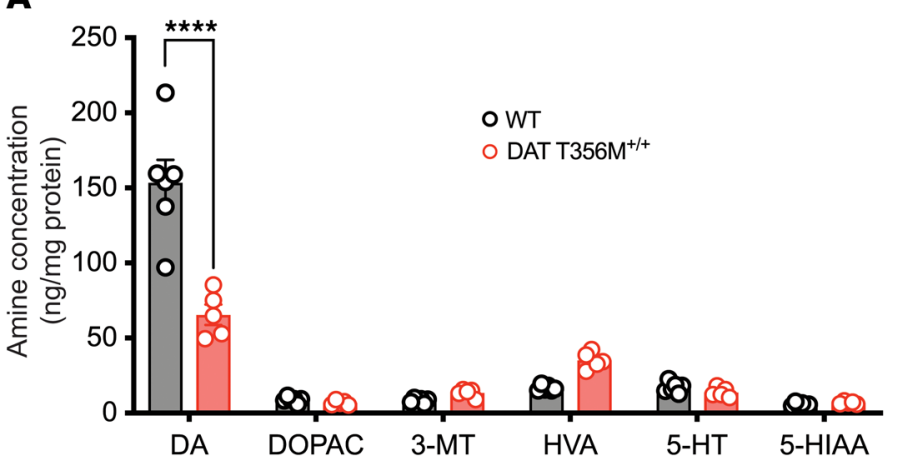

C

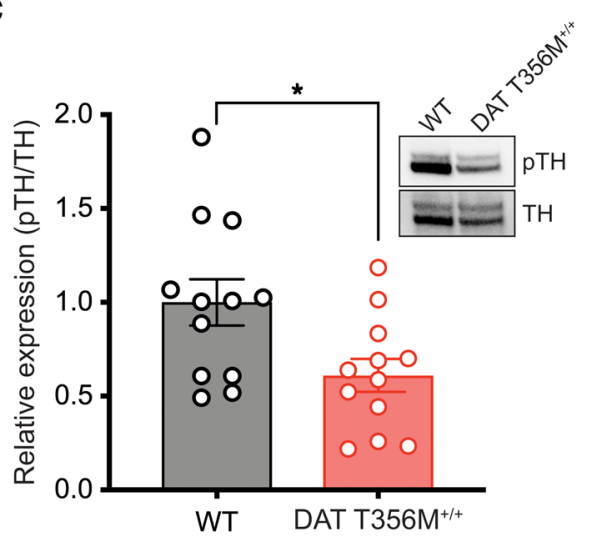

D

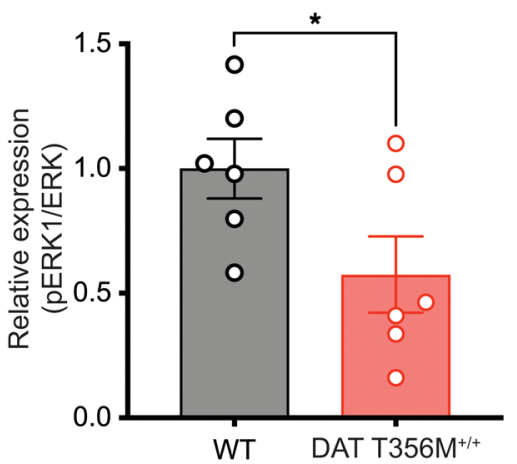

B

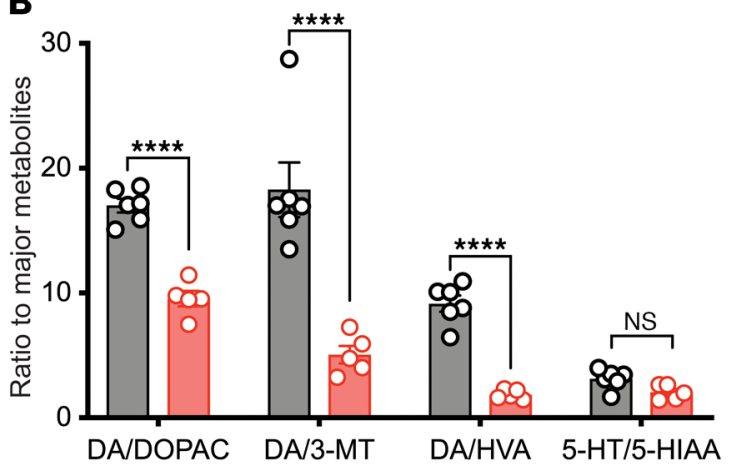

E

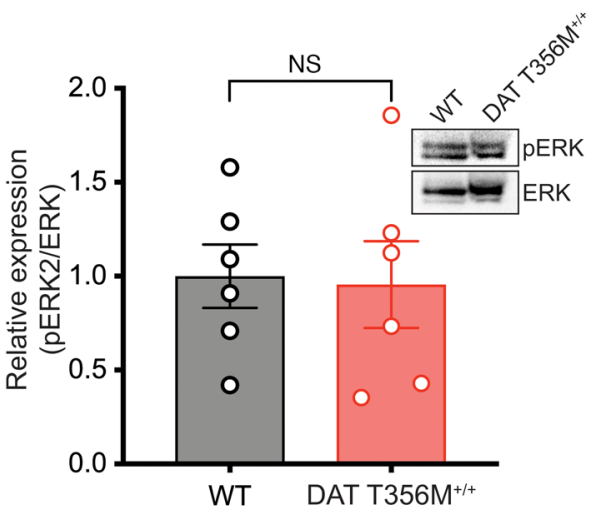

Figure 2. The DAT T356M mutation drives increased striatal DA metabolism and reduced striatal DA synthesis. (A) The tissue concentration of DA (measured by HPLC) is significantly reduced in the DAT T356M $/++$ striatum compared with WT striatum (WT = $153.5 \pm 15.38 \mathrm{ng} / \mathrm{mg}^{\circ}$ DAT T356M ${ }^{+/+}=65.55 \pm 6.71$ $\mathrm{ng} / \mathrm{mg} ; n=6$ WT, 5 DAT T356M ${ }^{++} ; P<0.0001$, 2-way ANOVA followed by Šidák's multiple comparisons test). There was no difference in the concentration of other biogenic amines (serotonin). ${ }^{* * *} P<0.0001$. (B) The ratio of the tissue content of DA in striatum to its metabolites is significantly lower in DAT $\mathrm{T}_{356 \mathrm{M}+/+}$ mice compared with WT mice (see Supplemental Table 2), providing evidence for increased metabolism of DA in the striatum (likely due to reduced reuptake of released DA). ${ }^{* * *} P<0.0001$. (C) Immunoblotting revealed significantly decreased pTH expression in the striatum of DAT T356M ${ }^{+/+}$mice when compared with WT mice ( $n=12$ from 4 animals; $P=0.0172$, Student's 2-tailed $t$ test). ${ }^{*} P=0.0172$. ( $\mathbf{D}$ and $\left.\mathbf{E}\right)$ Immunoblotting revealed significantly decreased p-ERK1 expression in the striatum of DAT T356M ${ }^{+/+}$mice when compared with WT mice $\left(n=6 ; P=0.03\right.$, Student's 2-tailed $t$ test). ${ }^{*} P=0.0279$ (D).

mice $\left(-\right.$ cocaine $[-\mathrm{COC}]: \mathrm{WT}=1.921 \pm 0.17 \mu \mathrm{M} ; \mathrm{DAT}$ T356M $\mathrm{M}^{+/+}=$ $1.526 \pm 0.18 \mu \mathrm{M} ; n=17 ; P=0.1908,2$-way ANOVA). These data indicate intact capacity of presynaptic dopaminergic terminals to properly release stored DA in DAT mutant animals. However, the decay time of the amperometric current $\left(t_{80}-t_{20}\right)$ was significantly greater in DAT T356 $\mathrm{M}^{+/+}$mice compared with WT mice (Figure 1C) (-COC: $\mathrm{WT}=121.5 \pm 12.39 \mathrm{~ms} ; \mathrm{DAT} \mathrm{T} 356 \mathrm{M}^{+/+}=244.9 \pm 19.83 ; n=17 ; P=$ 0.001, 2-way ANOVA followed by Šidák's multiple comparisons test). This increase reflects significantly reduced DAT-mediated DA clearance in DAT T356 $\mathrm{M}^{+/+}$mice. We then sought to determine whether the T356M mutation alters basal extracellular DA concentration in the striatum. We performed in vivo fast-scanning adsorption voltammetry (FSCAV) to measure basal DA levels (39). Basal values from a 20-minute collection period, capable of demonstrating stability, were used to calculate an average basal concentration for each animal. The basal concentration of extracellular DA was significantly higher in the striatum of DAT T356M $\mathrm{M}^{+/+}$animals compared with WT animals $\left(\mathrm{WT}=268.35 \pm 27.52 \mathrm{nM}\right.$; DAT T356M $\mathrm{M}^{+/+}$ $=359.54 \pm 28.67 \mathrm{nM} ; n=5 \mathrm{WT}, n=7 \mathrm{DAT}$ T356 $\mathrm{M}^{+/+} ; P=0.0453$, Welch's $t$ test). This increase in basal levels of DA provides evidence that the T356M mutation promotes elevated basal extracellular DA in the striatum, possibly by DAT-mediated anomalous DA efflux.
DAT is a known target for the psychoactive drug cocaine, which has been shown to impair DA reuptake from the synapse via competitive inhibition of the DAT $(40,41)$. To determine whether this mutation disrupted transporter sensitivity to cocaine (and to ensure our signal was dopaminergic), we measured release and reuptake dynamics of DA in the presence of $10 \mu \mathrm{M}$ cocaine in the striatum of WT and DAT T356 $\mathrm{M}^{+/+}$mice. Cocaine did significantly decrease the peak of the amperometric current in the DAT $\mathrm{T}_{356 \mathrm{M}^{+/+}}$mice compared with WT animals (Figure 1B) (+COC: $\mathrm{WT}=1.766 \pm 0.19 \mu \mathrm{M} ; \mathrm{DAT} \mathrm{T} 356 \mathrm{M}^{+/+}=0.916 \pm 0.13 \mu \mathrm{M} ; n=17$; $P=0.23$ and $P<0.001$, respectively, 2-way ANOVA followed by Šidák's multiple comparisons test). This decrease in the presence of cocaine might be due to an increase in buffering capacity for DA at the synapse mediated by DAT. One possibility for this observation is that DAT T356M has a decreased affinity for cocaine (38), therefore increasing the availability of DAT to bind DA upon release. Notably, cocaine significantly increased the decay time of the DA signal in both WT and DAT T356 $\mathrm{M}^{+/+}$mice (Figure 1C) $\left(+\mathrm{COC} ; \mathrm{WT}=334.1 \pm 25.82 \mathrm{~ms} ; \mathrm{DAT} \mathrm{T} 356 \mathrm{M}^{+/+}=384.9 \pm 32.21 \mathrm{~ms}\right.$; $n=17 ; P<0.001$ and $P<0.006$, respectively, 2-way ANOVA followed by Šidák's multiple comparisons test). These data indicate that (a) the mutant transporter does retain sensitivity to cocaine; 
A

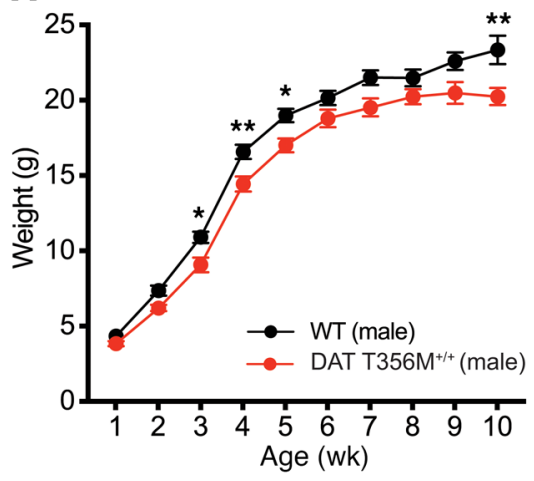

B

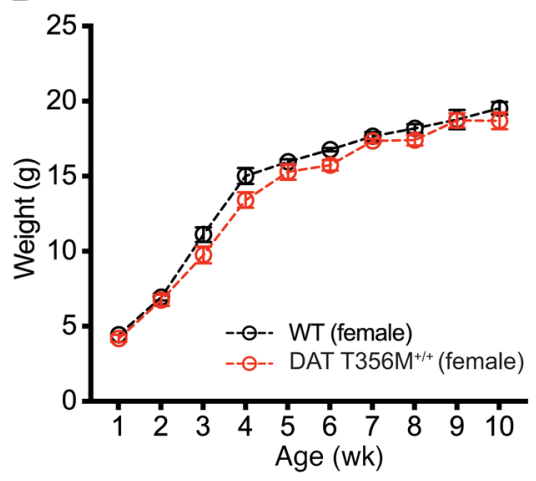

Figure 3. DAT $\mathrm{T} 56 \mathrm{M}^{+/+}$male, but not female, mice exhibit slower weight gain in early life and have lower body weights in adulthood than DAT WT male mice. (A) Male DAT T356M $\mathrm{M}^{+/+}$mice gain weight significantly more slowly in the period following weaning (P21 - week 5 of life; $n=37$ WT, 39 DAT

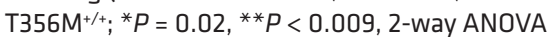
followed by Šidák's multiple comparisons test). In adulthood (at 10 weeks of age), the body weight of male DAT T356M ${ }^{+/+}$mice averaged $3.09 \mathrm{~g} \pm 0.92 \mathrm{~g}$ lower than that of adult male WT mice. (B) Female DAT T356M+/+ mice never differed statistically in body weight compared with WT female mice. (b) the amperometric signal is fine-tuned by DAT activity; and (c) the mutant transporter is capable of DA reuptake, although to a lesser extent.

The observed decreased DA clearance in DAT T356 $\mathrm{M}^{+/+}$mice could stem from either impairment of the DAT (i.e., reduced function or anomalous DA efflux) or from decreased expression. To determine whether DAT expression was decreased in DAT $\mathrm{T}^{2} 56 \mathrm{M}^{+/+}$mice, we used immunoblotting to quantify DAT expression relative to that in WT animals. We found no difference in DAT expression in the striatum between DAT T356 $\mathrm{M}^{+/+}$mice and WT animals (Figure 1D) $(n=5 ; P=0.877$, Student's 2-tailed $t$ test). These data suggest that the DAT T356M mutation impairs DA clearance by directly reducing transporter function and not by altering DAT availability. However, it should be noted that these data do not exclude the possibility of altered DAT expression at the plasma membrane.

DAT T356 $\mathrm{M}^{+/+}$mice have increased striatal DA metabolism and decreased tyrosine hydroxylase phosphorylation at Ser31. Based on our finding that the DAT T356M mutation reduces the rate of DA clearance, we sought to determine whether this resulted in increased DA metabolites and altered DA synthesis in the stria-
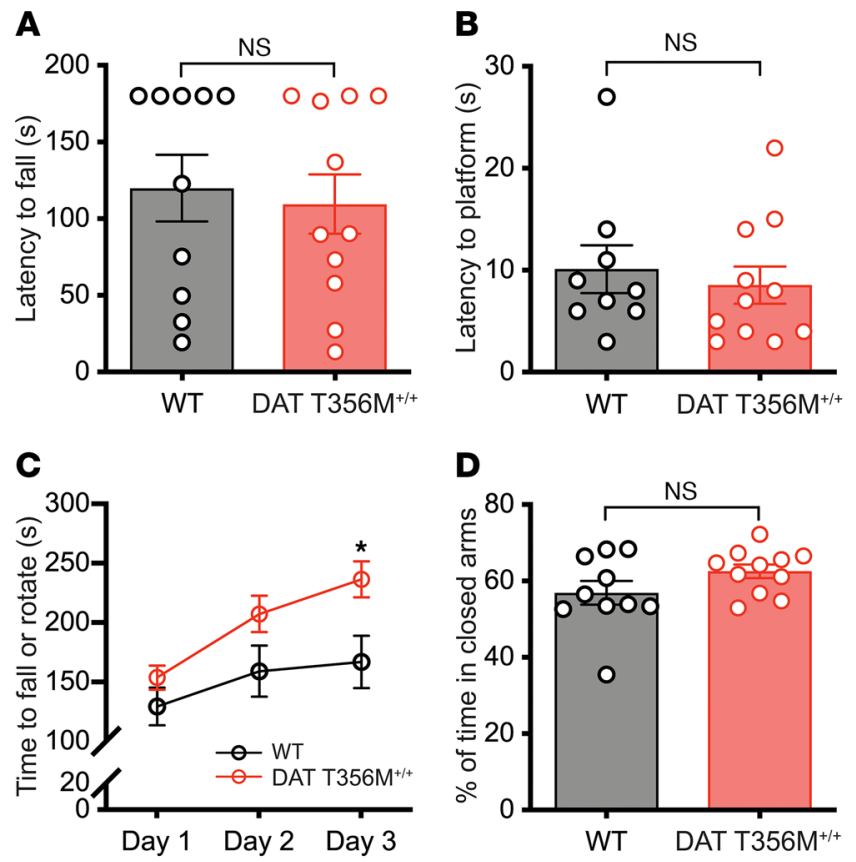

tum. We measured the striatal tissue content of biogenic amines and their metabolites in WT and DAT T356 $\mathrm{M}^{+/+}$mice. The concentration of DA was greatly reduced in DAT $\mathrm{T}^{2} 56 \mathrm{M}^{+/+}$mice compared with that WT mice (Figure $2 \mathrm{~A})(\mathrm{WT}=153.5 \pm 15.38 \mathrm{ng} /$ mg; DAT T356 $\mathrm{M}^{+/+}=65.55 \pm 6.71 \mathrm{ng} / \mathrm{mg} ; n=6 \mathrm{WT}, n=5 \mathrm{DAT}$ T356M ${ }^{+/+} ; P<0.0001,2$-way ANOVA followed by Šidák's multiple comparisons test). Moreover, the concentration of DA relative to its metabolites was significantly reduced in the striatum of DAT $\mathrm{T}_{356 \mathrm{M}^{+/+}}$mice compared with WT mice (Figure $2 \mathrm{~B}$ ) ( $n=6 \mathrm{WT}$, $n=5 \mathrm{DAT}$ T356 $\mathrm{M}^{+/+}$; see Supplemental Table 2; supplemental material available online with this article; https://doi.org/10.1172/ JCI127411DS1), supporting increased metabolism of DA in the striatum of DAT $\mathrm{T} 356 \mathrm{M}^{+/+}$mice. In contrast, there was no difference in serotonin (5-HT) content or in the ratio of 5-HT to its metabolite 5 -hydroxyindoleacetic acid (5-HIAA). To determine the impact of altered DA metabolism in the DAT T356 $\mathrm{M}^{+/+}$mouse on DA synthesis, we measured the expression of the rate-limiting enzyme, tyrosine hydroxylase (TH), and its phosphorylated (i.e., active) form. The activity of TH is regulated, in part, by phosphorylation of the residue Ser31 (42). Phosphorylation at this site results in increased activity of TH and, as a consequence, increased DA synthesis (42).

Figure 4. DAT T356 $\mathrm{M}^{+/+}$mice do not demonstrate deficits in strength, coordination, motor learning, or anxiety. (A) There was no difference in latency to fall on the inverted screen test (a proxy for strength) between WT and DAT T356M ${ }^{+/+}$mice $\left(\mathrm{WT}=120.0 \pm 21.79 \mathrm{~s}\right.$, DAT T356M ${ }^{+/+}=109.6$ $\pm 19.29 \mathrm{~s} ; n=10 \mathrm{WT}, n=11$ DAT T356M ${ }^{++} ; P=0.72$, Student's 2-tailed $t$ test). (B) There was no difference in latency to reach the platform on the pole climb test (a proxy for coordination) between WT and DAT T356M ${ }^{+/+}$ mice (WT $=10.11 \pm 2.360 \mathrm{~s}$, DAT T356M ${ }^{+/+}=8.545 \pm 1.836 \mathrm{~s} ; n=9 \mathrm{WT}, 11$ DAT T356M $\mathrm{M}^{+/+} ; P=0.6$, Student's 2 -tailed $t$ test). (C) On days 1 and 2 of the rotarod test of coordination and motor learning, there was no statistically significant difference in performance between WT and DAT T356M ${ }^{+/+}$mice. However, on the third day of testing, DAT T356 $\mathrm{M}^{+/+}$took a significantly longer time to fall or rotate than WT mice, indicating improved motor learning and indicating a propensity for the formation of repetitive motor routines in DAT T356M+/+mice (WT days 1, 2, $3=129.43 \pm 15.89 \mathrm{~s}, 159.06$ $\pm 21.52 \mathrm{~s}, 167.03 \pm 22.1 \mathrm{~s}$, respectively; DAT T356M ${ }^{+/+}$days 1, 2, $3=153.73 \pm$ $10.16 \mathrm{~s}, 207.36 \pm 15.28 \mathrm{~s}, 236.45 \pm 15.22 \mathrm{~s}$, respectively; $n=10 \mathrm{WT}, n=11$ DAT

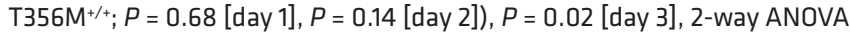
followed by Šidák's multiple comparisons test). (D) There was no difference in the percentage of time spent in the closed arms of the elevated zero maze between WT and DAT T356 $\mathrm{M}^{+/+}$mice, indicating no anxiety-like phenotype in the DAT T356M ${ }^{+/+}$mice $\left(\mathrm{WT}=56.94 \% \pm 3.12 \%\right.$, DAT T356M $\mathrm{M}^{++}$ $=62.55 \% \pm 1.75 \% ; n=10 \mathrm{WT}, n=11$ DAT T356M ${ }^{++} ; P=0.13$, Student's 2-tailed $t$ test). ${ }^{*} P=0.0157$. 
A

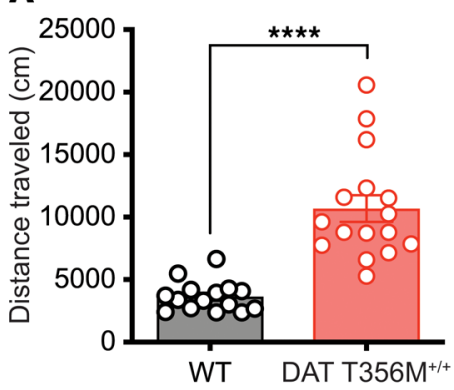

B
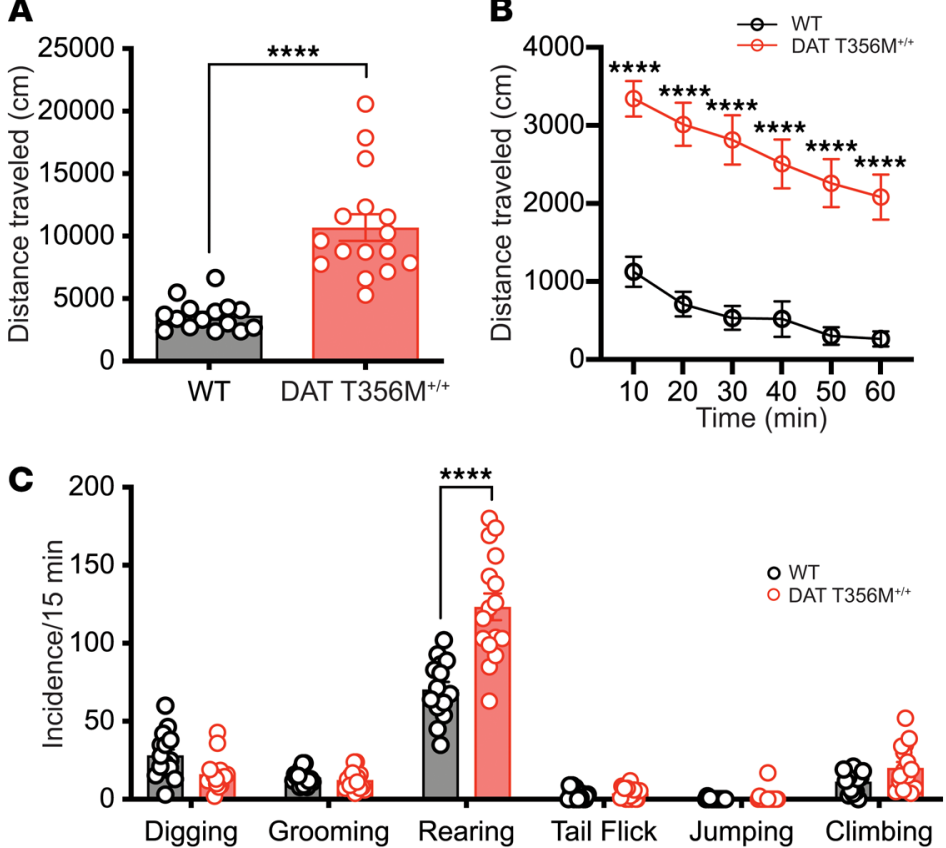

D
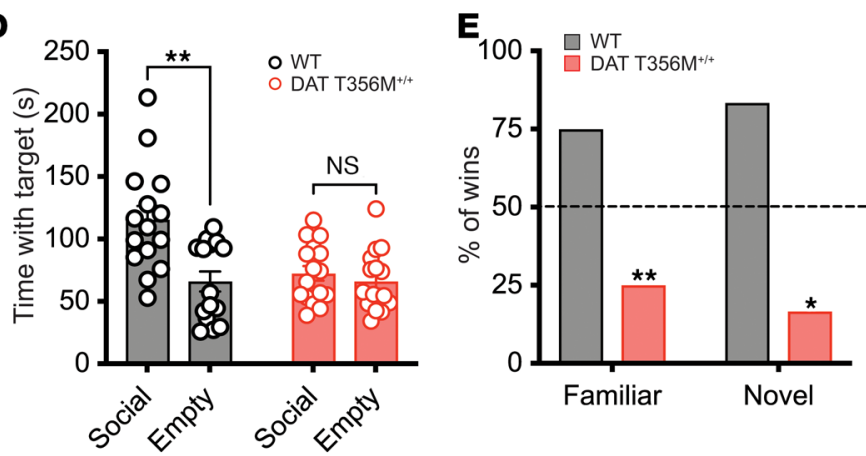

Figure 5. DAT T356M $\mathrm{M}^{+/+}$mice exhibit spontaneous, persistent hyperlocomotion, repetitive rearing behavior, and altered social behaviors. (A) DAT T356M+/+ mice traveled significantly further than WT mice during a 60-minute test period (WT = $3648 \pm 312.4$ $\mathrm{cm} ;$ DAT T356M $\mathrm{M}^{+/+}=20571 \pm 1062 \mathrm{~cm} ; n=15 \mathrm{WT}, 16$ DAT T356M+/+; ${ }^{* * * *} P<0.0001$, Student's 2-tailed $t$ test). (B) DAT T356M ${ }^{+/+}$mice exhibited hyperlocomotor activity across all 10-minute intervals of the 60 -minute test (for all intervals, ${ }^{* * *} P<0.0001$ by 2 -way ANOVA followed by Šidák's multiple comparison test). (C) DAT

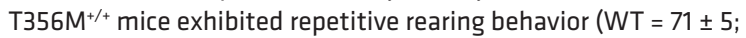
DAT T356M ${ }^{+/+}=123 \pm 9 ; n=15$ WT, $n=16$ DAT T356M ${ }^{+/+} ;{ }^{* * *} P<$ 0.0001, 2-way ANOVA followed by Šidák's multiple comparisons test). (D) WT animals displayed a statistically significant preference for the social target, while DAT T356M ${ }^{+/+}$mice exhibited no preference for either target $(\mathrm{WT}=115.41 \pm 11.06 \mathrm{~s}$ with social target, $66.07 \pm 8.12$ s with empty chamber; $n=15 ; P=0.001$, 2-way ANOVA followed by Šidák's multiple comparisons test; DAT T356M ${ }^{+/+}=72.51$ $\pm 5.91 \mathrm{~s}$ with social target, $66.125 \pm 5.97 \mathrm{~s}$ with empty chamber; $n=$ 16; $P=0.81$, 2-way ANOVA followed by Šidák's multiple comparisons test). ${ }^{*} P=0.0012$. (E) DAT T356M ${ }^{+/+}$mice won significantly fewer bouts against both familiar and novel mice than would be expected by chance (dashed line indicates chance-level performance; $n=24$ bouts from 12 pairs of mice; ${ }^{*} P<0.05,{ }^{* *} P<0.01$ by $\chi^{2}$ ).
Ser31 is phosphorylated by ERK1/2 (43), which is coupled to the DA $D_{2}$ autoreceptor $\left(D_{2} R\right)$ (44). Therefore, we measured changes in phosphorylation of TH in Ser31 (pTH Ser31) and found that pTH Ser31 was reduced in DAT $\mathrm{T} 356 \mathrm{M}^{+/+}$mice relative to WT mice (Figure 2C) ( $n=12$ from 4 animals for each genotype; $P=0.0172$, Student's 2-tailed $t$ test), with no change in total TH expression. Decreased TH phosphorylation could be a result of continuous stimulation of the $\mathrm{D}_{2} \mathrm{R}$ due to a reduced DA clearance in the DAT $\mathrm{T} 356 \mathrm{M}^{+/+}$mouse, which has been shown to decrease $\mathrm{D}_{2} \mathrm{R}$ function $(45,46)$. It is also consistent with previous literature demonstrating reduced TH Ser31 phosphorylation as well as increased extracellular DA levels in animals with loss of the DAT (46). Decreased $\mathrm{D}_{2} \mathrm{R}$ function would in turn result in reduced ERK1/2 activation and, therefore, reduced TH phosphorylation at Ser31. Reduced phosphorylation of TH at Ser31 would result in reduced DA synthesis. This is consistent with our HPLC data, which demonstrated reduced total tissue content of DA.

To determine whether ERK1/2 activation was indeed reduced in DAT T356 $\mathrm{M}^{+/+}$mice, we measured expression of phosphorylated ERK1/2 (p-ERK1/2) (its activated form). We found reduced p-ERK1 (but not p-ERK2) striatal expression relative to total ERK (Figure 2, $\mathrm{D}$ and $\mathrm{E} ; n=6 ; P=0.03$, Student's 2-tailed $t$ test), indicating down- regulation of ERK1 activity in the striatum. This is consistent with our finding of reduced phosphorylation of $\mathrm{TH}$ at Ser31.

Reduced body weight in DAT T356 $\mathrm{M}^{+/+}$male mice. Male DAT $\mathrm{T}_{356} \mathrm{M}^{+/+}$mice gained weight significantly more slowly than WT mice in the period immediately following weaning through the fifth week of life (Figure 3A) (P21-wk 5; $n=37$ WT, 39 DAT T356 $\mathrm{M}^{+/+} ; P=0.02, P<0.009,2$-way ANOVA followed by Šidák's multiple comparisons test). In adulthood (at 10 weeks of age), the body weight of male DAT T356 $\mathrm{M}^{+/+}$mice averaged $3.09 \mathrm{~g}( \pm 0.92$ g) lower than that of adult male WT mice. Conversely, female DAT $\mathrm{T}_{356} \mathrm{M}^{+/+}$body weights never differed significantly from those of WT mice (Figure 3B; $n=36 \mathrm{WT}, 28 \mathrm{DAT}$ T356 $\mathrm{M}^{+/+}$). As observed in DAT homozygous KO animals, slower weight gain in early life may be associated with impaired food intake (18) (however, caloric intake was not directly measured) or with increased activity (see the following section).

DAT T356 $\mathrm{M}^{+/+}$mice are hyperactive and exhibit repetitive behaviors and social deficits. As dysregulated DA homeostasis and signaling have been associated with movement disorders and anxiety, we first sought to determine whether $\mathrm{T} 356 \mathrm{M}$ produced deficits in strength and motor coordination and promoted anxiety-like behaviors. These behavioral traits were assessed prior to other 

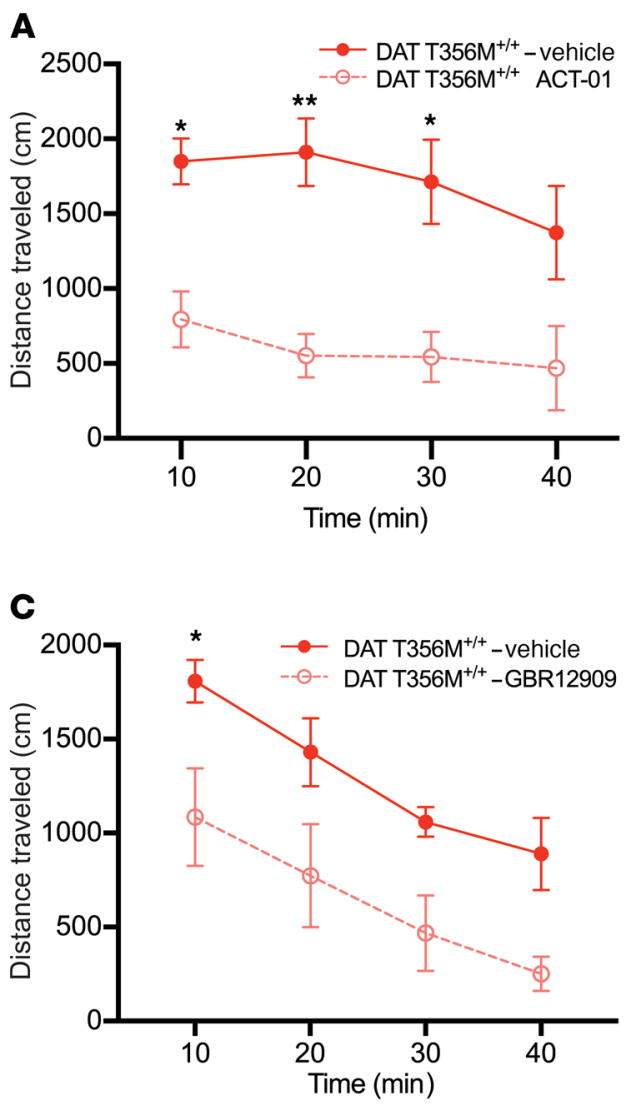

B

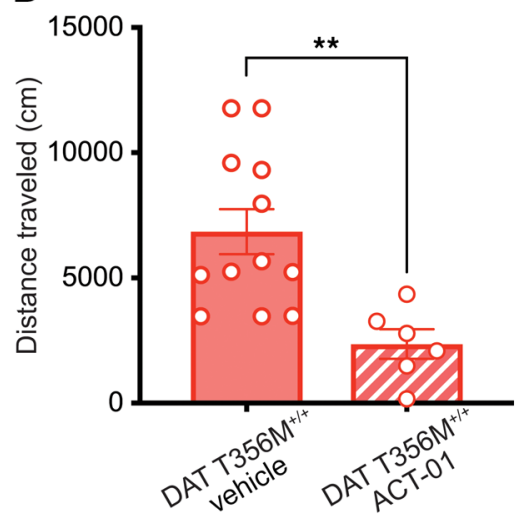

D

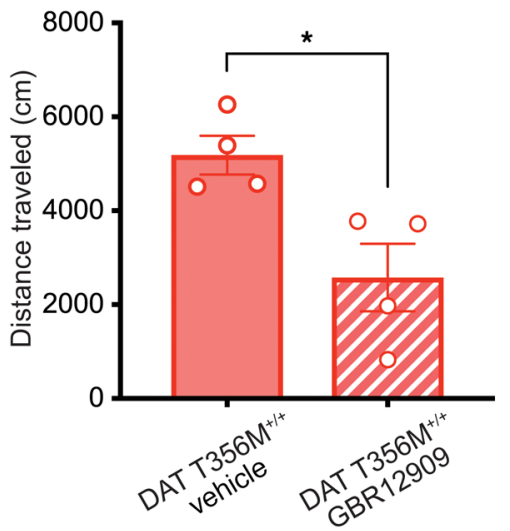

Figure 6. Treatment with DAT inhibitors reduces spontaneous locomotor activity in DAT T356 $\mathrm{M}^{+/+}$ mice. (A) ACT-01 treatment significantly reduced spontaneous locomotor activity in DAT T356M ${ }^{+/+}$ mice as early as 20 minutes into the observation period compared with vehicle-treated animals ( $n=6-9 ;{ }^{*} P=0.0032 ;{ }^{*} P<0.032,2$-way ANOVA followed by Šidák's multiple comparisons test).

(B) Total locomotor activity in DAT T356M ${ }^{+/+}$mice was reduced by acute treatment with ACT-01 $\left(\right.$ DAT T356M ${ }^{+/+}$vehicle $=6847 \mathrm{~cm} \pm 901.2 \mathrm{~cm}$; DAT

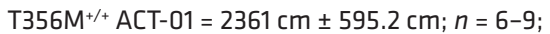
${ }^{*} P=0.0045$, Student's $t$ test). (C) GBR12909 treatment significantly reduced spontaneous locomotor activity in DAT T356M ${ }^{+/+}$mice as early as 10 minutes into the observation period compared with vehicle-treated animals $(n=4$; ${ }^{*} P=0.045,2$-way ANOVA followed by Šidák's multiple comparisons test). (D) Total locomotor activity in DAT T356M $\mathrm{M}^{+/+}$mice was rescued by acute treatment with GBR12909 (DAT T356M ${ }^{+/+}$ vehicle $=5188 \mathrm{~cm} \pm 411.7 \mathrm{~cm}$; DAT T356M ${ }^{+/+}$ CBR12909 $=2578 \mathrm{~cm} \pm 716.5 \mathrm{~cm} ; n=4 ;{ }^{*} P=$ 0.0196 , Student's 2-tailed $t$ test). testing, as these behaviors (if present) would complicate interpretation of other behavioral findings (see below). DAT T356 M $\mathrm{M}^{+/+}$ animals exhibited no motor strength or coordination deficits (as measured by inverted screen, pole climb, and rotarod). For all behavioral tests in the present work, both male and female animals were used. As there was no effect of sex in any of the behavioral tasks, male and female animals were combined for all analyses. DAT $\mathrm{T} 356 \mathrm{M}^{+/+}$animals had no difference in the following: time to fall on inverted screen (Figure $4 \mathrm{~A})(\mathrm{WT}=120 . \pm 21.79 \mathrm{~s}$, DAT $\mathrm{T}_{356} \mathrm{M}^{+/+}=109.6 \pm 19.29 \mathrm{~s} ; n=10 \mathrm{WT}, n=11 \mathrm{DAT} \mathrm{T}^{2} 56 \mathrm{M}^{+/+} ; P=$ 0.72 , Student's 2-tailed $t$ test); time to reach the platform on pole climb (Figure 4B) $\left(\mathrm{WT}=10.11 \pm 2.360 \mathrm{~s}, \mathrm{DAT} \mathrm{T} 356 \mathrm{M}^{+/+}=8.545 \pm\right.$ $1.836 \mathrm{~s}, n=9 \mathrm{WT}, n=11 \mathrm{DAT}$ T356 $\mathrm{M}^{+/+} ; P=0.6$, Student's 2-tailed $t$ test); or time to fall on rotarod, except on the third day of rotarod, on which day the DAT T356 $\mathrm{M}^{+/+}$mice outperformed the WT (Figure 4C) (WT: days 1, 2, 3, respectively $=129.43 \pm 15.89 \mathrm{~s}, 159.06$ $\pm 21.52 \mathrm{~s}, 167.03 \pm 22.1 \mathrm{~s} ;$ DAT T356M $\mathrm{M}^{+/+}$: days 1 , 2, 3, respectively $=153.73 \pm 10.16 \mathrm{~s}, 207.36 \pm 15.28 \mathrm{~s}, 236.45 \pm 15.22 \mathrm{~s} ; n=10 \mathrm{WT}, n$ $=11$ DAT T356M ${ }^{+++} ; P=0.68$, day $1 ; P=0.14$, day $2 ; P=0.02$, day 3, 2-way ANOVA followed by Šidák's multiple comparisons test). Additionally, we observed no anxiety phenotype as measured by the elevated zero maze test (Figure $4 \mathrm{D})(\mathrm{WT}=56.94 \pm 3.12 \%$, DAT $\mathrm{T}_{356} \mathrm{M}^{+/+}=62.55 \% \pm 1.75 \% ; n=10 \mathrm{WT}, n=11 \mathrm{DAT}$ T356M $\mathrm{M}^{+/+}$; $P=0.13$, Student's 2-tailed $t$ test).

We then sought to determine whether the T356M mutation promoted behaviors that have been associated with ASD or with the ADHD clinical phenotype, a common comorbidity of ASD. We tested animals for hyperactivity, social preference and dominance, and repetitive behaviors. We found that spontaneous locomotor activity was significantly elevated in DAT T356 $\mathrm{M}^{+/+}$mice (Figure $5 \mathrm{~A})\left(\mathrm{WT}=3648 \pm 312.4 \mathrm{~cm} ;\right.$ DAT T356M $\mathrm{M}^{+/+}=20571 \pm 1062 \mathrm{~cm}$; $n=15 \mathrm{WT}, n=16$ DAT T356 $\mathrm{M}^{+/+} ; P<0.0001$, Student's 2-tailed $t$ test). These animals traveled, on average, over 5 times the distance of their WT littermates in the same period of time (60 minutes). This hyperactivity persisted for the duration of the test (Figure 5B) (for all bins, $P<0.0001$ by 2 -way ANOVA followed by Šidák's multiple comparison test). To assess whether DAT $\mathrm{T}_{356} \mathrm{M}^{+/+}$mice exhibited repetitive behaviors, we video monitored the animals for 15 minutes and quantified episodes of specific behaviors (self-grooming, climbing on wire cage lid, rearing, tail flick, jumping, and digging) (Figure 5C; see Supplemental Table 3). We observed that DAT T356 $\mathrm{M}^{+/+}$mice exhibited repetitive rearing behavior, performing this specific behavior nearly twice as often as their WT littermates $\left(\mathrm{WT}=71 \pm 5\right.$; DAT $\mathrm{T} 356 \mathrm{M}^{+/+}=123 \pm 9$; $n=15 \mathrm{WT}, n=16 \mathrm{DAT} \mathrm{T}^{2} 6 \mathrm{M}^{+/+} ; P<0.0001,2$-way ANOVA followed by Šidák's multiple comparisons test). There was no difference in the frequency of self-grooming or digging behaviors, although a trend toward reduced digging was observed in DAT $\mathrm{T} 356 \mathrm{M}^{+/+}$mice (Figure $\left.5 \mathrm{C}\right)\left(\mathrm{WT}=28 \pm 4\right.$; DAT T356 $\mathrm{M}^{+/+}=16 \pm 3$; $n=15 \mathrm{WT}, n=16$ DAT T356M ${ }^{+/+} ; P=0.0773$, 2-way ANOVA followed by Šidák's multiple comparisons test). There was also no difference in episodes of tail flick or jumping, both of which can be used as indicators of stress and anxiety. As the striatum has been implicated in action selection (the task of resolving con- 
flicts between competing behavioral alternatives) (47, 48), we used marble burying to determine the impact of this mutation on the capacity for action selection and propensity for repetitive behaviors in DAT T356 $\mathrm{M}^{+/+}$. We found that DAT $\mathrm{T} 356 \mathrm{M}^{+/+}$mice buried significantly fewer marbles than WT mice $(\mathrm{WT}=39.02 \% \pm$ $7.95 \%$ of marbles buried, DAT T356 $\mathrm{M}^{+/+}=8.26 \% \pm 2.97 \%$ of marbles buried; $n=15$ WT, $n=16$ DAT T356M ${ }^{+/+} ; P=0.0009$, Student's 2-tailed $t$ test), which can be interpreted to indicate a loss of motivated behavior or altered action selection. We next tested DAT $\mathrm{T}^{2} 6 \mathrm{M}^{+/+}$mice for deficits in social interactions using the 3 -chamber test of sociability (49). In this test, DAT T356 $\mathrm{M}^{+/+}$mice exhibited a loss of preference for social novelty (spending equal time in the empty chamber and the chamber containing a social partner), a behavior associated with the ASD clinical phenotype (Figure 5D) $(\mathrm{WT}=115.41 \pm 11.06 \mathrm{~s}$ with social target, 66.07 \pm 8.12 s with empty chamber; $n=15 ; P=0.001,2$-way ANOVA followed by Šidák's multiple comparisons test; DAT $\mathrm{T}^{2} 56 \mathrm{M}^{+/+}=72.51$ $\pm 5.91 \mathrm{~s}$ with social target, $66.125 \pm 5.97 \mathrm{~s}$ with empty chamber; $n=16 ; P=0.81$, 2-way ANOVA followed by Šidák's multiple comparisons test). Finally, we used the tube test to further investigate the effect of the DAT T356M mutation on normal social behaviors. Our results demonstrate a loss of social dominance (indicative of a social deficit) in DAT $\mathrm{T} 356 \mathrm{M}^{+/+}$mice against both familiar and novel social stimulus mice (Figure $5 \mathrm{E} ; n=24$ bouts from 12 pairs of mice; $P<0.05$ by $\chi^{2}$ ). This pattern of behavioral alterations suggests the T356M mutation, via impairments of the transporter function, results in altered DA homeostasis that contributes to an ASD-associated phenotype, including aberrant social behaviors and repetitive behaviors. This mutation also drives hyperactivity, a behavior associated with ADHD, which is a common comorbidity of $\operatorname{ASD}(50,51)$ and which has been linked to DAT mutation $(11,23)$. In contrast to the observed differences between homozygous and WT animals, there were no significant differences between WT mice and DAT $\mathrm{T} 356 \mathrm{M}^{+/-}$mice in any of the behavioral paradigms tested.

DAT antagonists decrease hyperlocomotion in DAT T356M ${ }^{+/+}$ mice. Our group has demonstrated that the DAT T356M mutation drives anomalous DA efflux that is prevented by exposure to DAT blockers (12). We therefore hypothesized that blockade of the DAT by a specific DAT antagonist (ACT-01 [DRI Biosciences Corporation]; see Supplemental Table 4) would prevent DA efflux through the mutant transporter, thereby reducing the hyperlocomotion observed in DAT $\mathrm{T} 356 \mathrm{M}^{+/+}$mice. ACT-01 was selected, as this compound specifically blocks the DAT without off-target interactions with the norepinephrine or serotonin transporters (see Supplemental Table 4). Specificity of ACT-01 for the DAT was determined by RTI-55 displacement as well as inhibition of $\left[{ }^{3} \mathrm{H}\right]$ DA uptake, $\left[{ }^{3} \mathrm{H}\right] 5-\mathrm{HT}$ uptake, and $\left[{ }^{3} \mathrm{H}\right] \mathrm{NE}$ uptake in HEK293 cells expressing, respectively, the human DAT (hDAT), the human serotonin transporter (hSERT), and the human norepinephrine transporter (hNET) (see Supplemental Table 4 for Kis, Hill coefficients, and $\mathrm{IC}_{50} \mathrm{~s}$ ). This allowed us to directly assess the impact of DAT blockade on behavior. ACT-01 treatment significantly reduced hyperlocomotion as early as 20 minutes into the observation period (Figure $6 \mathrm{~A})(n=6-9 ; P=0.0032$ at 20 minutes; $P<0.032$ at 30 minutes, 2-way ANOVA followed by Šidák's multiple comparisons test). Total locomotor activity was also reduced by acute treat- ment with ACT-01 (Figure 6B) $\left(\right.$ DAT T356M ${ }^{+/+}$vehicle $=6847 \mathrm{~cm}$ $\pm 901.2 \mathrm{~cm} ;$ DAT T356M ${ }^{+/+}$ACT-01 $=2361 \mathrm{~cm} \pm 595.2 \mathrm{~cm} ; n=6-9$; $P=0.0045$, Student's $t$ test). Due to the recent identification of this compound, we also used a previously validated DAT antagonist, GBR12909, to further support our findings. Treatment with GBR12909 significantly reduced spontaneous locomotor activity in DAT T356 $\mathrm{M}^{+/+}$mice in the first 10 minutes of observation (Figure $6 \mathrm{C} ; n=4 ; P=0.045$, 2-way ANOVA followed by Šidák's multiple comparisons test). Total locomotor activity was also reduced by acute treatment with GBR12909 (Figure 6D) $\left(\mathrm{DAT}\right.$ T356M ${ }^{+/+}$ vehicle $=5188 \mathrm{~cm} \pm 411.7 \mathrm{~cm} ;$ DAT T356M ${ }^{+/+}$GBR12909 $=2578$ $\mathrm{cm} \pm 716.5 \mathrm{~cm} ; n=4 ; P=0.0196$, Student's 2-tailed t test). These data support our hypothesis that anomalous DA efflux, mediated by the mutant DAT, leads to hyperlocomotion, which is corrected by blockade of the DAT.

\section{Discussion}

The study of DA and the proteins that regulate this neurotransmitter system (i.e., the DAT) are of critical importance to elucidating the role of DA dysfunction in health and disease. DAT variants, dysregulation of DA, and altered developmental trajectory of dopaminergic structures have been associated with ASD and related neuropsychiatric disorders, such as ADHD (11-13, 23, 37, 52, 53). By studying discrete variations of the DAT, we can not only further understand the impact of these variants on transporter function, but also determine how transporter dysfunction contributes to neurophysiological and behavioral alterations. Here, we present what we believe is the first murine model of an ASD-associated de novo mutation to the DAT, the DAT T356M mutation. The DAT T356M model allows us to explore both the physiological and phenotypic consequences of this mutation, particularly as it pertains to DA homeostasis and behaviors known to be DA dependent. We show here that DAT T356 $\mathrm{M}^{+/+}$mice exhibit patterns of behavior typically associated with the ASD phenotype (altered social interactions and repetitive behaviors) as well as behaviors associated with ADHD (i.e., hyperactivity), a common comorbidity of ASD. Our investigation links altered dopaminergic signaling due to DAT dysfunction caused by a genetic variant associated with ASD with behavioral alterations that recapitulate aspects of the human disorder in a murine model.

At the physiological level, our studies demonstrate that the DAT T356M mutation drives reduced transporter function, synaptic hyperdopaminergia, and reduced DA synthesis in the striatum. Impaired transporter function (specifically, reduced rate of DA reuptake from the extracellular space) aligns with previous work in transfected cells demonstrating reduced capacity of DAT T356M for DA reuptake and the presence of anomalous DA efflux through these defective transporters $(37,38)$. Although the expression of the T356M DAT is comparable to that of WT DAT, the possibility exists that altered DAT trafficking promoted by the T356M mutation drives impaired DA clearance. We believe this to be unlikely, given previous work demonstrating intact surface expression of the mutant transporter in cultured cell lines. Furthermore, the amperometric data presented here demonstrate reduced (but not eliminated) transporter function that is sensitive to cocaine, suggesting the presence of the transporter on the membrane. Our work strongly supports a mechanism whereby the reduced rate 
of DAT-mediated reuptake of released DA from the extracellular space results in $\mathrm{D}_{2} \mathrm{R}$ desensitization, reduced $\mathrm{DA}$ synthesis as a result of elevated synaptic DA levels, and ultimately, reduced total tissue content of DA. These results align with previous work in DAT KO animals, which demonstrates that desensitization of the $\mathrm{D}_{2} \mathrm{R}$ leads to reduced phosphorylation (activation) of $\mathrm{TH}$, the rate-limiting enzyme in the DA synthesis pathway $(46,54)$.

It is now understood that dopaminergic signaling in the striatum governs action selection (the task of resolving conflicts between competing behavioral alternatives) (55-57). Under physiological conditions, the DA signal is tightly regulated to support a quantitative prediction error signal (a physiological correlate of reward encoded by dopaminergic neurons as the difference between the current reward and the predicted reward based on previous experience) (20). This prediction error signal is critical to reward processing and learning of motivated behaviors $(20,21,58,59)$. Evidence presented here strongly supports a mechanism whereby transporter dysfunction drives dysregulation of the dopaminergic signal. It follows, therefore, that change in the nature of the DA signal (here, alteration in the phasic and quantitative nature of the signal due to transporter dysfunction) would lead to alterations in motivated behaviors (such as social behaviors). Here, we show that DAT T356M $\mathrm{M}^{+/+}$ animals have deficits in social behaviors, as demonstrated by loss of social preference in the 3-chamber task and loss of social dominance in the tube test. This supports our hypothesis that the DAT T356M drives aberrant, ASD-associated behaviors, which may be due, at least in part, to altered social motivation due to the underlying alteration in dopaminergic signaling. Furthermore, the work presented here demonstrates deficits in marble burying in DAT $\mathrm{T}_{356 \mathrm{M}^{+/+}}$mice. Marble burying represents an innate behavior of laboratory mice that is motivated by investigation (60). A deficit in marble burying can therefore be interpreted to represent a change in motivated behavior in mice. Our finding of reduced marble burying in DAT T356 $\mathrm{M}^{+/+}$ mice therefore supports our hypothesis that DAT dysfunction leading to synaptic hyperdopaminergia drives altered action selection and motivated behavior. Moreover, impulsivity and repetitive behaviors can be interpreted as deficits in inhibitory action control (61) and have been previously related to altered dopaminergic signaling $(16,62-64)$. The repetitive rearing behavior observed in DAT $\mathrm{T} 356 \mathrm{M}^{+/+}$mice further supports our hypothesis that dopaminergic dysregulation due to this mutation drives altered action selection and control. Finally, we demonstrate increased rotarod learning in DAT $\mathrm{T}_{356 \mathrm{M}^{+/+}}$mice. The superior performance of the DAT $\mathrm{T}^{2} 56 \mathrm{M}^{+/+}$animals on the rotarod test on the third day of testing provides evidence suggesting that this autism-associated mutation drives an increased propensity for the formation of repetitive behaviors (65) (a core diagnostic feature of ASD), presumably by altering DA homeostasis in the striatum (65). This enhanced learning on the rotarod task has been observed previously in multiple mouse lines with ASD-associated mutations and has been linked to altered DA neuron signaling.

In addition to the role of DA in action selection and reward learning, it has been shown that DA regulates spontaneous locomotor activity (66-69) and that hyperactivity can be driven in a
DA-dependent fashion (70). It follows therefore that enhanced synaptic DA levels in the striatum due to the DAT T356M mutation could drive hyperactivity. Indeed, our work demonstrates marked hyperactivity in DAT T356 $\mathrm{M}^{+/+}$mice. We further demonstrate the DAT dependence of this hyperactivity via blockade of the DAT, which reduced spontaneous locomotor activity in DAT $\mathrm{T}_{356 \mathrm{M}^{+/+}}$mice. This blockade was obtained both with the recently identified DAT blocker (ACT-01) and with a traditional DAT blocker (GBR12909). Taken together, these results suggest anomalous efflux of DA and reduced DA reuptake by the mutant transporter (both of which contribute to synaptic hyperdopaminergia) as driving factors in the observed hyperactivity. As increased locomotion was observed in flies expressing this variant in dopaminergic neurons and due to the high comorbidity of ASD and ADHD, we chose to explore the reversibility of the observed hyperlocomotion. Future work should aim to determine whether blockade of the DAT may eliminate or alleviate the more complex behavioral changes observed in DAT T356 ${ }^{+/+}$animals.

It is interesting to note that the DAT M356 variant was identified as a heterozygote in the proband (for patient genetic information and clinical evaluation, see supplemental material for Hamilton et al.; ref. 37). We found no evidence of behavioral or biochemical differences between WT and DAT T356 $\mathrm{M}^{+/-}$mice in our study. While much conservation of functional DNA sequences does exist between humans and mice, the sequences involved in transcriptional regulation in mice do diverge from their human orthologues and thus could provide a basis for differential expression profiles of various genes in mice (71), including the DAT. Differential (or preferential) expression profiles of the DAT T356 allele vs. the DAT M356 allele could serve as the foundation for the discrepancy between the observed human genotype-phenotype correlation and the one reported here. Alternatively, the possibility exists that the proband harbored a unique set of common variants that acted in concert with the DAT M356 variant allele to produce the observed phenotype. This presents an exciting new avenue for future research.

The behavioral and neurophysiological characteristics of the DAT T356M mouse presented here provide evidence for a role of DAT dysfunction (specifically anomalous DA efflux and reduced reuptake of released DA) in the behavioral deficits typically associated with ASD and ADHD. This work supports and extends previous in vitro work that demonstrated anomalous DA efflux and reduced uptake of DA resulting from this mutation. Our model suggests that blockade of the DAT may improve behavior through reduction of nonvesicular, DAT-mediated DA efflux. Given the number of roles of DA in behavioral learning, reward processing, and action selection, this work presents the exciting possibility of a potential mechanistic underpinning (in at least a subset of patients) for the altered behaviors observed in ASD and ADHD. Future work should seek to determine to what degree DA dysfunction exists across patients with these neuropsychiatric disorders and whether striatal dysfunction plays a central role in the pathology of these conditions.

\section{Methods}

Male and female mice maintained on a C57BL/6 background were used for all studies. All animals were derived from matings of DAT T356 $6^{+-}$parents. 


\section{Antibodies}

Details of primary and secondary antibodies used in this study are summarized in Supplemental Table 1.

\section{Generation of DAT T356M+/+ mice}

Mice were generated by GenOway S.A. The point mutation was inserted into the exon 8 of the mouse Slc $6 a 3$ gene and was expressed under the control of the endogenous Slc6a3 promoter. It should be noted that the mouse orthologue of the T356M variant is T355M. The T356M terminology will be used in the manuscript to refer to this mutation to maintain consistency with the nomenclature used in previous studies referring to this variant. A neomycin-positive selection cassette flanked by loxP sites was inserted into intron 7 in a region devoid of experimentally validated regulatory regions and of predicted transcription factor binding sites conserved between mouse and human. Isolated homology fragments were used to generate the targeting vector. PCR validation was used to confirm the recombination over the $3^{\prime}$ homology arm. Southern blot was used to validate recombination over the $3^{\prime}$ and $5^{\prime}$ homology arms. PCR and Southern blot were used to validate recombinase-mediated excision. After linearization to enhance homologous recombination, embryonic stem (ES) cells were electroporated with the targeting vector and subjected to positive selection. The presence of the correct recombination event was validated by PCR and Southern blot. Recombined ES cell clones, derived from black-coated C57BL/6 mice, were injected into blastocysts, derived from an albino C57BL/6 mouse strain, which were then implanted in pseudo-pregnant females to produce chimeric males carrying the combined locus. Chimerism was assessed by coat color marker comparison. Male mice with more than $50 \%$ chimerism were bred with C57BL/6 mice ubiquitously expressing the Cre-recombinase to excise the lox P flanked neomycin selection cassette and to generate heterozygous mice carrying the neo-excised point mutant knockin allele. Progeny were genotyped by PCR, and the recombinase-mediated excision event was validated by Southern blot. Heterozygous animals identified by PCR screening and Southern blot were investigated by sequencing analysis to confirm the integration of the mutation.

\section{Chronoamperometry}

DA release and reuptake were measured using chronoamperometry in striatal slices. Striatal hemislices $(300 \mu \mathrm{m})$ from 6- to 10-week-old WT and DAT T356M ${ }^{+/+}$mice were prepared with a Vibratome (Leica VT1000S) in an ice-cold oxygenated $\left(95 \% \mathrm{O}_{2} / 5 \% \mathrm{CO}_{2}\right)$ aCSF solution consisting of the following: $125 \mathrm{mM} \mathrm{NaCl}, 2.5 \mathrm{mM} \mathrm{KCl}, 1 \mathrm{mM} \mathrm{MgCl}_{2}$, $2 \mathrm{mM} \mathrm{CaCl}_{2}, 1.2 \mathrm{mM} \mathrm{NaH}_{2} \mathrm{PO}_{4}, 10 \mathrm{mM}$ dextrose, $26 \mathrm{mM} \mathrm{NaHCO}_{3}$, and $0.25 \mathrm{mM}$ ascorbic acid. Slices were then recovered in an oxygenated NMDG-HEPES bath (consisting of the following: $100 \mathrm{mM}$ NMDG, $2.5 \mathrm{mM} \mathrm{KCl}, 1.2 \mathrm{mM} \mathrm{NaH} \mathrm{PO}_{4}, 30 \mathrm{mM} \mathrm{NaHCO}, 20 \mathrm{mM}$ HEPES, $25 \mathrm{mMglucose}, 10 \mathrm{mMMgSO}_{4} \cdot 7 \mathrm{H}_{2} \mathrm{O}, 0.5 \mathrm{mMCaCl}_{2} \cdot 2 \mathrm{H}_{2} \mathrm{O}, 5 \mathrm{mML}$-ascorbic acid, $3 \mathrm{mM}$ sodium pyruvate, $2 \mathrm{mM}$ thiourea, $12 \mathrm{mM} \mathrm{N}$-acetyl-Lcysteine adjusted to $\mathrm{pH} 7.3-7.4$ ) for 12 minutes at $32-34^{\circ} \mathrm{C}$. Slices were then moved to an oxygenated aCSF bath (as above) at $28^{\circ} \mathrm{C}$ for a minimum of 1 hour prior to recording. Carbon fiber electrodes were advanced angularly into the desired recording site in the striatum so that the tip of the electrode was positioned at a depth of $75-100 \mu \mathrm{m}$ beneath the tissue surface. DA release was stimulated by a $100 \mathrm{~ms} 100-200 \mu \mathrm{A}$ potential pulse delivered by a bipolar electrode while the carbon fiber electrode measured DA release and clearance dynamics. Data were collected with an Axopatch 200B amplifier. Decay time was calculated as time for the current to decay from $80 \%$ of peak current to $20 \%$ of peak current $\left(t_{80}-t_{20}\right)$. For these experiments, $n=17$ slices for each genotype from 6 animals of each genotype.

\section{Voltammetry}

FSCAV was used to measure basal DA levels in the striatum. Mice between the ages of 8 and 16 weeks old were anesthetized with an i.p. injection of urethane $(25 \%[\mathrm{w} / \mathrm{v}]$ in sterile saline) at a volume of $7 \mu \mathrm{L}$ per $1 \mathrm{~g}$ body weight. A heating pad from Braintree Scientific was used to maintain mouse body temperature $\left(37^{\circ} \mathrm{C}\right)$ throughout the duration of the experiment. Stereotaxic surgery was performed with coordinates determined with reference to bregma, according to Franklin and Paxinos (72). A pseudo $\mathrm{Ag} / \mathrm{AgCl}$ reference was placed in the contralateral hemisphere. A stainless-steel stimulation electrode (diameter $0.2 \mathrm{~mm}$, MS303/2-A/SPC; Plastics One) was lowered into the medial forebrain bundle. A $50 \mu \mathrm{m}$ nafion-coated carbon fiber microelectrode (CFM), made as previously described (39), served as the working electrode and was lowered in the striatum. FSCAV was performed using instrumentation and software (WCCV 3.05) developed by Knowmad Technologies LLC. DA presence was confirmed by first collecting FSCV files. A 30-second file was collected and a stimulation applied at 5 seconds ( $60 \mathrm{~Hz}$ biphasic, $360 \mu \mathrm{A}, 120$ pulse stimulation, $2 \mathrm{~ms}$ per phase) through a linear constant current stimulus isolator (NL800A Neurolog; Digitimer Ltd.) to evoke DA release. The release was measured at the CFM in the striatum by applying a DA triangular waveform $(-0.4 \mathrm{~V}$ to $1.4 \mathrm{~V}$ to $-0.4 \mathrm{~V}$ scan rate $400 \mathrm{~V} / \mathrm{s})$. Once the presence of DA had been confirmed, FSCAV was applied for ambient DA concentration measurements. A DA FSCAV waveform was applied as previously described (39). Signals collected were processed with WCCV 3.05 software using LabVIEW 2009, which included signal deconvolution, filtering, and smoothing. The cyclic voltammogram (CV) at the third scan (following the controlled adsorption period) was extracted to integrate the DA oxidation peak between approximately $0.45 \mathrm{~V}$ and 0.9 V. Calibration curves were acquired prior to experiments and plotted as charge, in $\mathrm{pC}$, versus DA concentration. A separate calibration set ( $n=5$ electrodes) was obtained using both pre- and postcalibrations to determine the factor by which the sensitivity of the electrode changes after the experiment. Each precalibration was then adjusted by this factor and used to calculate in vivo values that were specific to each electrode. Following data collection, a large voltage was applied to the electrode ( $10 \mathrm{~V}$ for $45 \mathrm{~s})$ to lesion the tissue. Mice were subsequently euthanized via cervical dislocation followed by decapitation, and the brain was removed from the skull and stored in $4 \%$ paraformaldehyde in PBS solution. Prior to sectioning, the brain was transferred into a $30 \%$ sucrose solution until it was saturated with the medium. The brain was then flash-frozen, sectioned into $20 \mu \mathrm{m}$ slices mounted onto frosted glass slides, and the lesion identified to confirm the electrode was placed in the striatum.

\section{Western blot}

Mice were decapitated under isoflurane anesthesia at 7 to 10 weeks of age. The brain was rapidly dissected and placed in ice-cold sucrose solution consisting of the following: $210 \mathrm{mM}$ sucrose, $20 \mathrm{mM} \mathrm{NaCl}$, $2.5 \mathrm{mM} \mathrm{KCl}, 1.2 \mathrm{mM} \mathrm{NaH}_{2} \mathrm{PO}_{4}, 1 \mathrm{mM} \mathrm{MgCl}, 26 \mathrm{mM} \mathrm{NaHCO}_{3}$, and $10 \mathrm{mM}$ dextrose. The striatum was then dissected on ice and snapfrozen. Dissected tissue was homogenized in $200 \mu \mathrm{L}$ of homoge- 
nization buffer (RIPA buffer [Thermo Fisher Scientific], cOmplete protease inhibitor cocktail [MilliporeSigma], phosphatase inhibitor cocktail 3 [MilliporeSigma], $1 \mathrm{mM}$ sodium orthovanadate) on ice. Protein concentrations of all samples were determined by a bicinchoninic acid (BCA) protein assay using the Pierce BCA Protein Assay Kit (Thermo Fisher Scientific, 23225). Samples were resolved using NuPAGE Bis-Tris precast gels (Thermo Fisher Scientific, 10\% 10-well, NP0301BOX). The Precision Plus Protein Kaleidoscope Prestained Ladder was run alongside samples for protein size reference. Proteins were transferred from gels onto nitrocellulose membranes using the iBlot system (Thermo Fisher Scientific, IB23001). Western blot analysis was performed using antibodies listed in Supplemental Table 1 and visualized using Western Lightning Plus ECL. Adobe Photoshop software was used to crop full blots. Western blots were quantified using the NIH ImageJ gel analysis tool. See complete unedited blots in the supplemental material.

\section{HPLC}

Measures of the concentration of biogenic amines were obtained by the Neurochemistry Core Facility at Vanderbilt University. The striatum was collected from animals 7 to 10 weeks old in the same manner as described for Western blots and stored at $-80^{\circ} \mathrm{C}$. Frozen brain tissue was homogenized using a tissue dismembrator (Misonix XL-2000, Qsonica) in 100-750 $\mu \mathrm{l}$ of 0.1M TCA, which contains $10^{-2}$ M sodium acetate, $10^{-4} \mathrm{M}$ EDTA, and $10.5 \%$ methanol ( $\mathrm{pH}$ 3.8). Ten microliters of homogenate were used for protein assay. Protein concentration was determined using the BSA Protein Assay Kit (Thermo Scientific). Then samples were spun in a microcentrifuge at 10,000 $g$ for 20 minutes. The supernatant was removed for biogenic monoamines analysis. Biogenic amine concentrations were determined utilizing an Antec Decade II (oxidation: 0.65) electrochemical detector operated at $33^{\circ} \mathrm{C}$. Twenty microliter samples of the supernatant were injected using a Water 2707 autosampler onto a Phenomenex Kintex C18 HPLC column $(100 \times 4.60 \mathrm{~mm}, 2.6 \mu \mathrm{m})$. Biogenic amines were eluted with a mobile phase consisting of $89.5 \% 0.1 \mathrm{M} \mathrm{TCA}, 10^{-2}$ M sodium acetate, $10^{-4} \mathrm{M}$ EDTA, and $10.5 \%$ methanol (pH 3.8). Solvent was delivered at $0.6 \mathrm{ml} / \mathrm{min}$ using a Waters 515 HPLC pump. Using this HPLC solvent, the following biogenic amines eluted in the following order: norepinephrine, epinephrine, DOPAC, DA, 5-HIAA, HVA, 5-HT, and 3-MT. Data acquisition was managed by Empower software (Waters Corp.). Isoproterenol (5 ng/mL) was included in the homogenization buffer for use as an internal standard to quantify the biogenic amines of interest.

\section{Weight measurements}

All mice not undergoing experimental manipulation (including behavioral testing) were weighed weekly beginning 7 days after birth (P7). Animals undergoing behavioral testing were excluded from weight measurements.

\section{Behavioral paradigms}

All behavioral experiments were performed in the Vanderbilt University Neurobehavioral Core Facility. All behavioral tests were performed at the same time of day (13:00-17:00) using mice 8 to 10 weeks of age. Mice were group housed (3-5 mice/cage) on a 12-hour light/12-hour dark cycle with food and water available ad libitum. Mice were housed with corncob bedding. Mice were transferred to testing rooms followed by a 30-minue acclimation period before the start of each test. Unless otherwise stated, each apparatus used was cleaned with a $10 \%$ ethanol solution between each animal or trial to provide a standardized testing environment.

Behavior observations. Mice were placed individually into clean cages (containing bedding only) and recorded using a video camera for 15 minutes. Videos were then scored by observers blinded to genotype for the instances of the following behaviors: digging, grooming, rearing, tail flick, jumping, and climbing.

Elevated zero maze. Anxiety behaviors were assessed using an elevated zero maze $(62.5 \mathrm{~cm}$ outer diameter, $5 \mathrm{~cm}$ path width, $15 \mathrm{~cm}$ wall height, Stoelting). Animals were placed in center of the open portion of the maze at the start of each trial. Each trial was recorded by a video camera mounted to the ceiling, and analysis was performed using ANYmaze software (Stoelting). Data analyzed included the percentage of time in closed sections, number of entries into open and closed sections, and distance traveled in the maze.

Marble burying. Cages $(189 \mathrm{~mm} \times 297 \mathrm{~mm} \times 128 \mathrm{~mm})$ were prepared by filling clean, empty cages with $4.5 \mathrm{~cm}$ of Diamond Soft Bedding (Envigo Teklad 7089). Mice were placed individually into filled cages for a 5-minute acclimation period. Mice were then removed from the cages, and 15 blue marbles ( $15 \mathrm{~mm}$ diameter) cleaned with a $10 \%$ ethanol solution were gently placed in the cages. Marbles were evenly spaced in a $3 \times$ 5 configuration in each cage. A photograph was taken of each cage prior to returning mice to the cage and again 30 minutes later when mice were removed from the cage. Photographs of cages before and after testing were compared and scored by 3 independent, blinded observers. A marble was considered buried if more than two-thirds of the marble was covered with bedding at the end of 20 minutes.

Inverted screen. Mice were individually placed on a $7.5 \mathrm{~cm} \times 7.5 \mathrm{~cm}$ wire mesh screen that was mounted horizontally on a metal rod and elevated $40 \mathrm{~cm}$ above the apparatus floor. The rod was then slowly rotated $180^{\circ}$, ensuring all animals achieved a 4-paw grasp on the wire mesh before the apparatus was fully inverted. Time to fall was measured for each animal on 3 trials. The maximum time allowed was 180 seconds.

Horizontal pole climb. The horizontal climb tests the ability of a mouse to maintain balance on a horizontal rod (cloth tape wrapped, 1.5 $\mathrm{cm}$ diameter) suspended between 2 platforms. The pole is suspended $40 \mathrm{~cm}$ above the apparatus floor. The mouse was placed on the rod, and its movements were observed for 1 minute. Time to reach either platform or time to fall was measured for 3 trials for each mouse.

Open-field locomotion. Spontaneous locomotor activity in an open field was measured using $27 \times 27 \times 20.5$ chambers (Med Associates) placed within sound-attenuating boxes $(64 \times 45 \times 42 \mathrm{~cm})$ that were light and air controlled. Locomotion was detected by infrared beam disruption by the body of the mouse. Sixteen evenly spaced photocells located $1 \mathrm{~cm}$ above the chamber floor measured horizontal movement, while 16 photocells located $4 \mathrm{~cm}$ above the chamber floor captured vertical movements (jumping and rearing). Animals were placed in the activity chambers, and their activity was recorded for 60 minutes. Total distance traveled was analyzed using the Kruskal-Wallis test followed by Dunn's multiple comparison test (GraphPad Prism 8.0.2). Data from this test were also used to measure anxiety-like behaviors. Thigmotaxis, the tendency of the mice to remain close to the walls of the open field, can be used as an index of anxiety. Percentage of time mice spent in the periphery of the open field ( $\sim 52 \%$ of area) versus the center was determined. 
Rotarod. Rotarod was used to assess the balance and motor coordination of the mice on a rotating rod as well as the propensity for acquisition of repetitive motor routines (73). The rotarod apparatus consists of a rubber-covered cylindrical rod (3 $\mathrm{cm}$ in diameter) suspended $30 \mathrm{~cm}$ above the apparatus floor and separated into 5 equal compartments by plastic dividers with a radius of $15 \mathrm{~cm}$. The starting rotation rate was $4 \mathrm{rpm}$ and increased steadily to $40 \mathrm{rpm}$ over a period of 4 minutes. Mice were placed on the rod, and the timer was started. The time to fall or to complete 2 passive rotations was measured for each animal with a maximum time of 5 minutes. Animals completed 3 trials each day for 3 days. The time to fall or rotate for each day was calculated as an average over the time to fall or rotate for the 3 trials completed in a given day.

Tube test. The tube test was used to assess social dominance (a test for normal social behavior). One DAT T356M ${ }^{+/+}$knockin mouse and one WT mouse were placed head first at opposite ends of a clear plastic tube $(2.5 \mathrm{~cm}$ inner diameter, $13 \mathrm{~cm}$ length) and released simultaneously. Each trial ended when 1 mouse completely retreated from the tube or after 3 minutes. The mouse remaining in the tube was designated the winner and the retreating mouse was designated the loser. If neither animal retreated from the tube after 3 minutes, the trial was designated a draw. Each pair of animals participated in 2 trials, starting on alternating ends of the tube on each trial. A $\chi^{2}$ 1-sample analysis was used to determine whether the scores of the mutant mice were significantly different from an outcome expected by chance (50:50 win-lose).

Three-chamber test. The 3-chamber test was used to assess social preference (a test for normal social behavior in mice). The apparatus consists of a $60 \times 42 \times 22 \mathrm{~cm}$ box divided into 3 equal-sized compartments. Removable doors initially confined the test mouse to the center chamber, where the mouse habituated for 5 minutes. After 5 minutes, the doors were removed and the mouse was allowed to freely explore the apparatus. A stimulus mouse was then introduced in an inverted wire cup in one side chamber, while a clean, empty, inverted pencil cup was introduced in the opposite chamber. The stimulus mouse was always an age- and sex-matched WT mouse, either known to the subject mouse (familiar social stimulus) or unknown to the subject mouse (novel social stimulus). The subject mouse was then allowed to explore all 3 chambers freely for 10 minutes. A research assistant blinded to genotype then coded videos for time spent in each chamber and time spent interacting with the stimulus mouse or the empty inverted wire cup.

ACT-01 reversal of hyperactivity. Mice were allowed to habituate to the test room for 15 minutes prior to treatment. Mice naive to the openfield apparatus were treated with either $50 \mathrm{mg} / \mathrm{kg}$ of ACT- 01 or with vehicle via i.p. injection 30 minutes prior to testing. The same protocol for measuring spontaneous locomotor activity was then followed as is described under the Open-field locomotion section. ACT-01 was provided in-house.

GBR12909 reversal of hyperactivity. Mice were allowed to habituate to the test room for 15 minutes prior to treatment. Mice naive to the open-field apparatus were treated with either $20 \mathrm{mg} / \mathrm{kg}$ of GBR12909 or with vehicle via i.p. injection 30 minutes prior to testing. The same protocol for measuring spontaneous locomotor activity was then followed as is described under the Open-field locomotion section.

\section{Statistics}

All statistical analyses were performed using GraphPad Prism software (version 8.0.2). Statistical methods are indicated in the figure legends and Results. Data are presented as mean \pm SEM. Differences are considered statistically significant at $P<0.05$. Unpaired 2-tailed Student's $t$ test was used for 2-group comparisons, unless stated otherwise. Either 1- or 2-way ANOVA with Šidák's test was used for multiple comparisons.

\section{Study approval}

All behavioral, biochemical, and chronoamperometry experiments were performed under a protocol approved by the Vanderbilt University Animal Care and Use Committee. For FSCAV, a protocol approved by the Institutional Animal Care and Use Committees of the University of South Carolina was followed.

\section{Author contributions}

GED, UG, MTW, AG conceptualized the study. GED, JIA, FEH, $\mathrm{AW}$, and KEB carried out the experiments. GED, FEH, AW, PH, MTW, and AG conducted formal analyses of the data. UG and HC provided resources. FEH, JIA, HJGM, HC, FH, MR, and UG provided conceptual advice and edited the manuscript. MTW and AG acquired funding and supervised the study. GED, AG, and MTW prepared data and figures and wrote the manuscript. All authors contributed to the editing and review of the manuscript.

\section{Acknowledgments}

The authors would like to acknowledge the Vanderbilt Neurobehavioral Core (supported by NIH U54HD083211) and, in particular, Krista Paffenroth for assistance with behavioral data collection. We would also like to thank the Vanderbilt Neurochemistry Core (supported by the Vanderbilt Kennedy Center for Excellence in Developmental Disabilities, grant 90DD0595) for assistance with HPLC data collection. We would like to thank Saunders Consulting for editing the manuscript. Research reported in this publication was supported by NIH R01DA038058 (to AG), NIH R01DA35263 (to AG), NIH F30MH115535 (to GED), NIH F31MH114316 (to JIA), NIH R01MH106563 (to PH), and NIH T32GM007347. The content is solely the responsibility of the authors and does not necessarily represent the official views of the NIH.

Address correspondence to: Aurelio Galli, 1032 Tinsley Harrison Tower, 1900 University Boulevard, Birmingham, Alabama 35233, USA. Phone: 205.975.1932; Email: agalli@uab.edu. Or to: Mark Wallace, 465 21st Avenue South, MRB III Suite 7110, Nashville, Tennessee 37240, USA. Phone: 615.936.7108; Email: mark.wallace@vanderbilt.edu.
1. Doya K. Complementary roles of basal ganglia and cerebellum in learning and motor control. Curr Opin Neurobiol. 2000;10(6):732-739.

2. Schultz W. Dopamine neurons and their role in reward mechanisms. Curr Opin Neurobiol.
1997;7(2):191-197.

3. Nieoullon A. Dopamine and the regulation of cognition and attention. Prog Neurobiol. 2002;67(1):53-83.

4. Schultz W. Updating dopamine reward signals.
Curr Opin Neurobiol. 2013;23(2):229-238.

5. Albin RL, Young AB, Penney JB. The functional anatomy of basal ganglia disorders. Trends

Neurosci. 1989;12(10):366-375.

6. Surmeier DJ, Ding J, Day M, Wang Z, Shen W. 
D1 and D2 dopamine-receptor modulation of striatal glutamatergic signaling in striatal medium spiny neurons. Trends Neurosci. 2007;30(5):228-235.

7. Temlett JA. Parkinson's disease: biology and aetiology. Curr Opin Neurol. 1996;9(4):303-307.

8. Robinson TE, Berridge KC. The neural basis of drug craving: an incentive-sensitization theory of addiction. Brain Res Brain Res Rev. 1993;18(3):247-291.

9. Cousins DA, Butts K, Young AH. The role of dopamine in bipolar disorder. Bipolar Disord. 2009;11(8):787-806.

10. Tripp G, Wickens JR. Neurobiology of ADHD. Neuropharmacology. 2009;57(7-8):579-589.

11. Gadow KD, Roohi J, DeVincent CJ, Hatchwell E. Association of ADHD, tics, and anxiety with dopamine transporter (DAT1) genotype in autism spectrum disorder. JChild Psychol Psychiatry. 2008;49(12):1331-1338.

12. Bowton E, et al. SLC6A3 coding variant Ala559Val found in two autism probands alters dopamine transporter function and trafficking. Transl Psychiatry. 2014;4:e464.

13. Langen M, Bos D, Noordermeer SD, Nederveen $\mathrm{H}$, van Engeland H, Durston S. Changes in the development of striatum are involved in repetitive behavior in autism. Biol Psychiatry. 2014;76(5):405-411.

14. Anderson BM, et al. Examination of association to autism of common genetic variationin genes related to dopamine. Autism Res. 2008;1(6):364-369.

15. American Psychiatric Association. Neurodevelopmental Disorders: Autism Spectrum Disorder. Diagnostic and Statistical Manual of Mental Disorders. 5th ed. Washington, DC, USA: American Psychiatric Association; 2013.

16. Langen M, Durston S, Kas MJ, van Engeland $\mathrm{H}$, Staal WG. The neurobiology of repetitive behavior: ...and men. Neurosci Biobehav Rev. 2011;35(3):356-365.

17. Rodriguiz RM, Chu R, Caron MG, Wetsel WC. Aberrant responses in social interaction of dopamine transporter knockout mice. Behav Brain Res. 2004;148(1-2):185-198.

18. Giros B, Jaber M, Jones SR, Wightman RM, Caron MG. Hyperlocomotion and indifference to cocaine and amphetamine in mice lacking the dopamine transporter. Nature. 1996;379(6566):606-612.

19. Fiorillo CD, Song MR, Yun SR. Multiphasic temporal dynamics in responses of midbrain dopamine neurons to appetitive and aversive stimuli. JNeurosci. 2013;33(11):4710-4725.

20. Bayer HM, Glimcher PW. Midbrain dopamine neurons encode a quantitative reward prediction error signal. Neuron. 2005;47(1):129-141.

21. Schultz W. Dopamine reward prediction error coding. Dialogues Clin Neurosci. 2016;18(1):23-32.

22. Pessiglione M, Seymour B, Flandin G, Dolan RJ, Frith CD. Dopamine-dependent prediction errors underpin reward-seeking behaviour in humans. Nature. 2006;442(7106):1042-1045.

23. Cook EH, et al. Association of attention-deficit disorder and the dopamine transporter gene. Am J Hum Genet. 1995;56(4):993-998.

24. Gill M, Daly G, Heron S, Hawi Z, Fitzgerald M. Confirmation of association between attention deficit hyperactivity disorder and a dopamine transporter polymorphism. Mol Psychiatry. 1997;2(4):311-313.

25. Hansen FH, et al. Missense dopamine transporter mutations associate with adult parkinsonism and ADHD. J Clin Invest. 2014;124(7):3107-3120.

26. Kurian MA, et al. Homozygous loss-of-function mutations in the gene encoding the dopamine transporter are associated with infantile parkinsonism-dystonia. J Clin Invest. 2009;119(6):1595-1603.

27. Scott-Van Zeeland AA, Dapretto M, Ghahremani DG, Poldrack RA, Bookheimer SY. Reward processing in autism. Autism Res. 2010;3(2):53-67.

28. Sinha P, et al. Autism as a disorder of prediction. Proc Natl Acad Sci U S A. 2014;111(42):15220-15225.

29. Pogorelov VM, Rodriguiz RM, Insco ML, Caron MG, Wetsel WC. Novelty seeking and stereotypic activation of behavior in mice with disruption of the Dat1 gene. Neuropsychopharmacology. 2005;30(10):1818-1831.

30. Zhuang X, et al. Hyperactivity and impaired response habituation in hyperdopaminergic mice. Proc Natl Acad Sci U S A . 2001;98(4):1982-1987.

31. Rickhag M, et al. A C-terminal PDZ domain-binding sequence is required for striatal distribution of the dopamine transporter. Nat Commun. 2013;4:1580.

32. Mazei-Robison MS, Couch RS, Shelton RC, Stein MA, Blakely RD. Sequence variation in the human dopamine transporter gene in children with attention deficit hyperactivity disorder. Neuropharmacology. 2005;49(6):724-736.

33. Grünhage F, et al. Systematic screening for DNA sequence variation in the coding region of the human dopamine transporter gene (DAT1). Mol Psychiatry. 2000;5(3):275-282.

34. Mazei-Robison MS, et al. Anomalous dopamine release associated with a human dopamine transporter coding variant. J Neurosci. 2008;28(28):7040-7046.

35. Mergy MA, et al. The rare DAT coding variant Val559 perturbs DA neuron function, changes behavior, and alters in vivo responses to psychostimulants. Proc Natl Acad Sci U S A. 2014;111(44):E4779-E4788.

36. Neale BM, et al. Patterns and rates of exonic de novo mutations in autism spectrum disorders. Nature. 2012;485(7397):242-245.

37. Hamilton PJ, et al. De novo mutation in the dopamine transporter gene associates dopamine dysfunction with autism spectrum disorder. $\mathrm{Mol}$ Psychiatry. 2013;18(12):1315-1323.

38. Herborg F, Andreassen TF, Berlin F, Loland CJ, Gether U. Neuropsychiatric disease-associated genetic variants of the dopamine transporter display heterogeneous molecular phenotypes. J Biol Chem . 2018;293(19):7250-7262.

39. Atcherley CW, Wood KM, Parent KL, Hashemi P, Heien ML. The coaction of tonic and phasic dopamine dynamics. Chem Commun (Camb). 2015;51(12):2235-2238.

40. Beuming $\mathrm{T}$, et al. The binding sites for cocaine and dopamine in the dopamine transporter overlap. Nat Neurosci. 2008;11(7):780-789.

41. Huang X, Gu HH, Zhan CG. Mechanism for cocaine blocking the transport of dopamine: insights from molecular modeling and dynamics simulations. J Phys Chem B.
2009;113(45):15057-15066.

42. Dunkley PR, Bobrovskaya L, Graham ME, von Nagy-Felsobuki EI, Dickson PW. Tyrosine hydroxylase phosphorylation: regulation and consequences. J Neurochem. 2004;91(5):1025-1043.

43. Daubner SC, Le T, Wang S. Tyrosine hydroxylase and regulation of dopamine synthesis. Arch Biochem Biophys. 2011;508(1):1-12.

44. Dadalko OI, et al. mTORC2/rictor signaling disrupts dopamine-dependent behaviors via defects in striatal dopamine neurotransmission. J Neurosci. 2015;35(23):8843-8854.

45. Calipari ES, et al. Amphetamine self-administration attenuates dopamine D2 autoreceptor function. Neuropsychopharmacology. 2014;39(8):1833-1842.

46. Salvatore MF, Calipari ES, Jones SR. Regulation of tyrosine hydroxylase expression and phosphorylation in dopamine transporter-deficient mice. ACS Chem Neurosci. 2016;7(7):941-951.

47. Nonomura $S$, et al. Monitoring and updating of action selection for goal-directed behavior through the striatal direct and indirect pathways. Neuron. 2018;99(6):1302-1314.e5.

48. Seo M, Lee E, Averbeck BB. Action selection and action value in frontal-striatal circuits. Neuron. 2012;74(5):947-960.

49. Moy SS, et al. Sociability and preference for social novelty in five inbred strains: an approach to assess autistic-like behavior in mice. Genes Brain Behav. 2004;3(5):287-302.

50. Leyfer OT, et al. Comorbid psychiatric disorders in children with autism: interview development and rates of disorders. J Autism Dev Disord. 2006;36(7):849-861.

51. Simonoff E, Pickles A, Charman T, Chandler S, Loucas T, Baird G. Psychiatric disorders in children with autism spectrum disorders: prevalence, comorbidity, and associated factors in a population-derived sample. J Am Acad Child Adolesc Psychiatry. 2008;47(8):921-929.

52. Langen $\mathrm{M}$, et al. Changes in the developmental trajectories of striatum in autism. Biol Psychiatry. 2009;66(4):327-333.

53. LaHoste GJ, et al. Dopamine D4 receptor gene polymorphism is associated with attention deficit hyperactivity disorder. Mol Psychiatry. 1996;1(2):121-124.

54 . Jones SR, et al. Loss of autoreceptor functions in mice lacking the dopamine transporter. $\mathrm{Nat} \mathrm{Neu}$ rosci. 1999;2(7):649-655.

55. Mink JW. The basal ganglia: focused selection and inhibition of competing motor programs. Prog Neurobiol. 1996;50(4):381-425.

56. Redgrave P, Prescott TJ, Gurney K. The basal ganglia: a vertebrate solution to the selection problem? Neuroscience. 1999;89(4):1009-1023.

57. Cisek P, Kalaska JF. Neural mechanisms for interacting with a world full of action choices. Annu Rev Neurosci. 2010;33:269-298.

58. Lak A, Stauffer WR, Schultz W. Dopamine prediction error responses integrate subjective value from different reward dimensions. Proc Natl Acad Sci U S A. 2014;111(6):2343-2348.

59. Wise RA. Dopamine, learning and motivation. Nat Rev Neurosci. 2004;5(6):483-494.

60. Londei T, Valentini AM, Leone VG. Investigative burying by laboratory mice may involve non-functional, compulsive, behaviour. Behav 
Brain Res. 1998;94(2):249-254.

61. Colzato LS, van den Wildenberg WP, van Wouwe NC, Pannebakker MM, Hommel B. Dopamine and inhibitory action control: evidence from spontaneous eye blink rates. Exp Brain Res. 2009;196(3):467-474.

62. Voon V, Potenza MN, Thomsen T. Medicationrelated impulse control and repetitive behaviors in Parkinson's disease. Curr Opin Neurol. 2007;20(4):484-492.

63. Weintraub D, et al. Impulse control disorders in Parkinson disease: a cross-sectional study of 3090 patients. Arch Neurol. 2010;67(5):589-595.

64. Staal WG, de Krom M, de Jonge MV. Brief report: the dopamine-3-receptor gene (DRD3) is associated with specific repetitive behavior in autism spectrum disorder (ASD). J Autism Dev Disord.
2012;42(5):885-888.

65. Rothwell PE, et al. Autism-associated neuroligin-3 mutations commonly impair striatal circuits to boost repetitive behaviors. Cell. 2014;158(1):198-212.

66. Pijnenburg AJ, van Rossum JM. Letter: Stimulation of locomotor activity following injection of dopamine into the nucleus accumbens. JPharm Pharmacol. 1973;25(12):1003-1005.

67. Beninger RJ. The role of dopamine in locomotor activity and learning. Brain Res. 1983;287(2):173-196.

68. Fray PJ, Sahakian BJ, Robbins TW, Koob GF, Iversen SD. An observational method for quantifying the behavioural effects of dopamine agonists: contrasting effects of d-amphetamine and apomorphine. Psychopharmacology (Berl). 1980;69(3):253-259.
69. Cole SO. Brain mechanisms of amphetamineinduced anorexia, locomotion, and stereotypy: A review. Neurosci Biobehav Rev. 1978;2(2):89-100.

70. Pycock CJ, Horton RW. Dopamine-dependent hyperactivity in the rat following manipulation of GABA mechanisms in the region of the nucleus accumbens. J Neural Transm. 1979;45(1):17-33.

71. Yue F, et al. A comparative encyclopedia of DNA elements in the mouse genome. Nature. 2014;515(7527):355-364.

72. Franklin K, Paxinos G. The Mouse Brain in Stereotaxic Coordinates, Compact. Amsterdam, Netherlands: Elsevier; 2007.

73. Rothwell PE, et al. Autism-associated neuroligin-3 mutations commonly impair striatal circuits to boost repetitive behaviors. Cell. 2014;158(1):198-212. 